\title{
Removal of Perineuronal Nets Unlocks Juvenile Plasticity Through Network Mechanisms of Decreased Inhibition and Increased Gamma Activity
}

\author{
- Kristian Kinden Lensjø, ${ }^{1,3}$ - Mikkel Elle Lepperød, ${ }^{2,3}$ - Gunnar Dick, ${ }^{1}$ Torkel Hafting, ${ }^{2,3}$ and Marianne Fyhn ${ }^{1,3}$ \\ ${ }^{1}$ Department of Biosciences, ${ }^{2}$ Institute of Basic Medical Sciences, and ${ }^{3}$ Center for Integrative Neuroplasticity, University of Oslo, 0370 Oslo, Norway
}

Perineuronal nets (PNNs) are extracellular matrix structures mainly enwrapping parvalbumin-expressing inhibitory neurons. The assembly of PNNs coincides with the end of the period of heightened visual cortex plasticity in juveniles, whereas removal of PNNs in adults reopens for plasticity. The mechanisms underlying this phenomenon remain elusive. We have used chronic electrophysiological recordings to investigate accompanying electrophysiological changes to activity-dependent plasticity and we report on novel mechanisms involved in both induced and critical period plasticity. By inducing activity-dependent plasticity in the visual cortex of adult rats while recording single unit and population activity, we demonstrate that PNN removal alters the balance between inhibitory and excitatory spiking activity directly. Without PNNs, inhibitory activity was reduced, whereas spiking variability was increased as predicted in a simulation with a Brunel neural network. Together with a shift in ocular dominance and large effects on unit activity during the first $48 \mathrm{~h}$ of monocular deprivation (MD), we show that PNN removal resets the neural network to an immature, juvenile state. Furthermore, in PNN-depleted adults as well as in juveniles, MD caused an immediate potentiation of gamma activity, suggesting a novel mechanism initiating activity-dependent plasticity and driving the rapid changes in unit activity.

Key words: activity-dependent plasticity; cortical plasticity; gamma; inhibition; local field potential; perineuronal nets

\section{Significance Statement}

Emerging evidence suggests a role for perineuronal nets (PNNs) in learning and regulation of plasticity, but the underlying mechanisms remain unresolved. Here, we used chronic in vivo extracellular recordings to investigate how removal of PNNs opens for plasticity and how activity-dependent plasticity affects neural activity over time. PNN removal caused reduced inhibitory activity and reset the network to a juvenile state. Experimentally induced activity-dependent plasticity by monocular deprivation caused rapid changes in single unit activity and a remarkable potentiation of gamma oscillations. Our results demonstrate how PNNs may be involved directly in stabilizing the neural network. Moreover, the immediate potentiation of gamma activity after plasticity onset points to potential new mechanisms for the initiation of activity-dependent plasticity.

\section{Introduction}

During early postnatal development, cortical circuits are refined through experience to ensure adequate responses to stimuli from the outside world. This fine tuning relies on a high degree of plasticity, a phenomenon well described in the visual cortex

\section{Received Aug. 8, 2016; revised Nov. 22, 2016; accepted Dec. 1, 2016.}

Author contributions: K.K.L., M.E.L., G.D., T.H., and M.F. designed research; K.K.L., M.E.L., and M.F. performed research; K.K.L., M.E.L., and M.F. contributed unpublished reagents/analytic tools; K.K.L., M.E.L., and T.H. analyzed data; K.K.L., M.E.L., G.D., T.H., and M.F. wrote the paper.

This work was funded by the Research Council of Norway (Grant 217920 to M.F. and Grant 231248 to T.H) and the University of Oslo. We thank Rachel M. Thomas and Andrew Edwards for useful comments on the manuscript.

The authors declare no competing financial interests.

Correspondence should be addressed to Marianne Fyhn, Department of Biosciences, P.0. Box 1166 Blindern, University of 0slo, 0370 0slo, Norway. E-mail: marianne.fyhn@ibv.uio.no.

DOI:10.1523/JNEUROSCI.2504-16.2016

Copyright $\odot 2017$ the authors $\quad 0270-6474 / 17 / 371269-15 \$ 15.00 / 0$ through the classical model of ocular dominance (OD) plasticity (Wiesel and Hubel, 1963). A brief period of sensory deprivation by occluding one eye of vision causes a shift in OD and leads to life-long impairment of vision (amblyopia) if the occlusion is maintained beyond a certain age (Levelt and Hübener, 2012). The time window for heightened activity-dependent plasticity, or critical period (CP), is limited and depends on the maturation of inhibitory neurons after eye opening, in particular the maturation of the GABAergic fast-spiking parvalbumin $(\mathrm{PV}+)$ inhibitory neurons and their network integration (Fagiolini and Hensch, 2000; Fagiolini et al., 2004; Hensch, 2005; Southwell et al., 2010). Recent work suggests that the PV+ inhibitory neuron maturation suppresses spontaneous activity of excitatory neurons, thereby promoting visually evoked activity after eye opening (Toyoizumi et al., 2013). As the inhibitory activity reaches a 
plateau, the window for plasticity closes. This coincides with the assembly of stable extracellular matrix structures, the perineuronal nets (PNNs), which are formed mainly around the PV + neurons (Hockfield et al., 1990; Pizzorusso et al., 2002; Balmer et al., 2009; Carulli et al., 2010). At this stage, the network is stabilized and PV + neurons maintain a high activity state throughout adult life. Although the exact functions of the PNNs are not known, they may modulate the network's excitatory-inhibitory balance as several lines of evidence point to a regulatory role for PNNs in maintaining the high-activity state of PV + neurons (Beurdeley et al., 2012; Cabungcal et al., 2013; Donato et al., 2013; Liu et al., 2013; Morawski et al., 2015).

Enzymatic breakdown of PNNs by use of the bacterial enzyme chondroitinase $\mathrm{ABC}$ (chABC) in adult rodents induces juvenilelike plasticity in several brain regions, including OD plasticity in visual cortex, demonstrating a role for PNNs in restricting adult brain plasticity (Pizzorusso et al., 2002; Gogolla et al., 2009; Carulli et al., 2010; Happel et al., 2014). Nevertheless, how the removal of PNNs allows for increased plasticity remains elusive. In the present study, we investigated alterations in activity of inhibitory and excitatory neurons in vivo to reveal the functional relationship between plasticity regulation and inhibitory activity. By recording single units and field potential activity in primary visual cortex (V1) of freely moving adult and juvenile rats, we show for the first time that PNN removal in adults resets the neuronal network to an immature state with reduced inhibitory activity and high spiking variability similar to that of the CP. Increased spiking variability was also predicted from reducing inhibition in a Brunel neural network simulation.

To reveal the relationship between OD plasticity and changes in single unit activity, we recorded neural activity across time during a brief period of sensory deprivation using monocular deprivation (MD), both in adults after PNN removal and during the $\mathrm{CP}$ of juvenile animals. Although OD plasticity has been the canonical model for investigations of activity-dependent cortical plasticity, few studies have investigated how the neuronal network responds to MD across time using serial recordings in chronically instrumented animals (Mioche and Singer, 1989; Frenkel and Bear, 2004; Hengen et al., 2013; Rose et al., 2016). By recording the local field potentials and the activity of the very same units during the first $48 \mathrm{~h}$ of $\mathrm{MD}$, we observed remarkable functional changes in network responses. A pronounced and immediate increase in gamma activity was followed by changes in unit responses in the first $12-24 \mathrm{~h}$, suggesting that the changes are triggered by synchronous activity of $\mathrm{PV}+$ neurons in response to the imbalanced visual input. These data provide novel insight into the network mechanisms driving the initial changes of ocular dominance plasticity.

\section{Materials and Methods}

\section{Experimental animals}

Eighteen adult (4-6 months of age, 400-600 g) male Long-Evans rats were used for this study, of which 12 were used for electrophysiology and six were used for histology alone. In addition, three juvenile rats were implanted with tetrodes at postnatal day 22 (P22). Animals were bred locally and maintained at the animal facility at the Department of Biosciences at the University of Oslo. Animals were housed with a $12 \mathrm{~h}$ light/dark cycle (lights off at 8:00 A.M) and the experiments were performed in the dark phase. Animals used for electrophysiology were housed individually after surgery in transparent Perspex cages $(35 \times$ $55 \times 30 \mathrm{~cm}$ ) with ad libitum access to water and six to 10 food pellets per $24 \mathrm{~h}$. They were handled on a weekly basis from the age of 4-6 weeks and for $10-15$ min each of the $3 \mathrm{~d}$ before surgery. All experimentation was approved by the Norwegian Animal Research Committee before initiation.

\section{Tetrode and microdrive assembly}

Tetrodes were constructed from $17-\mu \mathrm{m}$-diameter platinum-iridium (90-10\%) wire (California Fine Wire). When the wires were intertwined, they were heated to fuse and the ends cut free. The heavy polyimide enamel insulation was removed at the outermost tips by holding the four free ends briefly over a lighter flame. Four tetrodes were assembled in a microdrive (Axona) and secured with conductive silver paint (HK Wentworth) and several layers of nail polish. Electrode tips were platinum coated before surgery to reduce the impedances to $150-200 \mathrm{k} \Omega$ at $1 \mathrm{kHz}$.

\section{Surgical procedures}

Protease-free chABC from Proteus vulgaris was purchased from Amsbio and diluted in filtered $1 \times$ PBS to a concentration of $0.05 \mathrm{U} / \mu \mathrm{l}$ (Pizzorusso et al., 2002). Glass pipettes with a $15-20 \mu \mathrm{m}$ opening diameter were backfilled with mineral oil, assembled into a NanoJect II micro injector (Drummond Scientific), and loaded with chABC.

Injections were made concomitantly with tetrode implantation at four sites in the binocular region of V1 (V1b) at two depths in each location (750 and $350 \mu \mathrm{m}$ below the dura). The locations were, relative to lambda, $0.5 \mathrm{~mm}$ anterior and $4.7 \mathrm{~mm}$ lateral, $0.5 \mathrm{~mm}$ anterior and $5.1 \mathrm{~mm}$ lateral, $0.5 \mathrm{~mm}$ posterior and $4.7 \mathrm{~mm}$ lateral, and $0.5 \mathrm{~mm}$ posterior and $5.0 \mathrm{~mm}$ lateral. A total volume of $3.1 \mu \mathrm{l}$ was injected in each hemisphere. The injections were made stepwise over $2 \mathrm{~min}$ at each position and the pipette was left for another $2 \mathrm{~min}$ before retraction.

All surgical procedures were performed in an aseptic environment. Rats were anesthetized with isoflurane mixed with air (5\% induction, $1.5-2 \%$ for maintenance) and immobilized in a stereotaxic frame (World Precision Instruments). They were given subcutaneous injections of buprenorphine $(0.04 \mathrm{mg} / \mathrm{kg})$ and local subcutaneous injections of bupivacaine/adrenaline (Marcain adrenaline, $13.2 \mathrm{mg} / \mathrm{kg}$ ) in the scalp before surgery began. The scalp was shaved and cleaned with ethanol and chlorhexidine. Heart rate and core temperature were monitored continuously throughout the operation using a MouseStat system (Kent Scientific), the latter in a feedback mechanism to a heating pad. In addition, the hindpaw withdrawal reflex was used to assess the depth of anesthesia.

Craniotomies of $1.5-2 \mathrm{~mm}$ in diameter were made bilaterally above the V1b based on the rat brain atlas (Paxinos and Watson, 2007) using a hand-held Perfecta-300 dental drill (W \& H Nordic). Tetrodes were implanted at AP $0 \pm 0.2 \mathrm{~mm}$ and ML $4.9 \pm 0.1 \mathrm{~mm}$ relative to lambda. The depth of implantation was $250-350 \mu \mathrm{m}$ measured from the surface of dura. Jeweler's screws fixed to the scull served as ground electrodes and the microdrives were secured to the scull using additional jeweler's screws and dental cement. All animals were given a subcutaneous injection of carprofen $(5 \mathrm{mg} / \mathrm{kg})$ at the end of the surgery, the edge of the wound was cleaned, and lidocaine ointment was applied. This was repeated for $3 \mathrm{~d}$ after surgery.

Animals that were used for histology alone underwent a similar procedure. After chABC injections, the craniotomies were filled with KwikSil silicone (World Precision Instruments) and the wound sutured shut. Medication was identical to the procedure described above.

\section{Electrophysiological recordings}

All recordings, unless described otherwise, were performed in awake, freely behaving animals. A total of 105 isolated units were recorded from four animals bilaterally injected with chABC and 122 units from three control animals. In addition, 138 isolated units were recorded from animals that underwent MD and 43 units were recorded from CP aged animals (P24-P26). The majority of recordings were from cells in layers $2 / 3$ and $4(250-750 \mu \mathrm{m}$ below dura). All units used for analyses of MD effects were recorded from layer $2 / 3$.

To allow the animals to recover fully from surgery, electrophysiological recordings were not initiated until $3 \mathrm{~d}$ after surgery. Activity was recorded in daily sessions for 7-10 d while the animals were presented with visual stimuli. Animals that underwent MD were treated separately, but were in a similar regime. In these animals, the MD was initiated no later than $5 \mathrm{~d}$ after surgery. The recording system used was daqUSB, 
provided by Axona. The microdrives, each carrying 16 electrodes in four tetrode configurations, were connected to a multichannel head stage and the animal placed in a $25 \times 25 \times 35 \mathrm{~cm}$ high glass box. The head stage was coupled through a lightweight multiwire cable to a preamplifier, which itself was coupled to the 64-channel recording unit. The Axona system also provides a digital amplifier in the recording software. Both analog and digital amplifiers were used in all of the experiments. A counterweight system was installed to relieve the extra weight of the cables and head stage from the animal to allow it to move freely inside the box. Data collection started when the signal amplitudes exceeded 4-5 times the noise level. If the signal was "silent"; that is, no cells were active, the tetrodes were lowered by $50 \mu \mathrm{m}$ and the animal connected again later the same day.

Signals were amplified 8000-15,000 times and band-pass filtered between 0.8 and $6.7 \mathrm{kHz}$. Triggered spikes were stored to disk at $48 \mathrm{kHz}$ (50 samples per waveform, 8 bits/sample) with a 32-bit time stamp (clock rate at $96 \mathrm{kHz}$ ). Spike waveforms above a threshold of $50 \mu \mathrm{V}$ were time stamped, digitized at $32 \mathrm{kHz}$ for $1 \mathrm{~ms}$, and saved to the hard drive for offline analysis. One channel in each hemisphere served as a reference electrode to record LFP; LFP signals were amplified 4000 times, low-pass filtered at $500 \mathrm{~Hz}$, and stored at $4.8 \mathrm{kHz}$ (16 bits/sample).

\section{Visual stimulation}

Visual stimulation was presented on four LCD computer monitors (Dell, $27.5 \times 34 \mathrm{~cm}, 60 \mathrm{~Hz}$ refresh rate) arranged in a square surrounding the glass box. The stimulus was created in MATLAB using the Psychophysics toolbox extensions (Brainard, 1997) and consisted of full-screen drifting sinusoidal gratings in 8 orientations of $3 \mathrm{~s}$ duration, at 100\% contrast, with a temporal frequency of $1 \mathrm{~Hz}$, spatial frequency of 0.08 , and a velocity of $25 \%$ s. The stimulus was repeated 6 times in a random order, interleaved by a $1 \mathrm{~s}$ blank gray screen (mean luminance) to estimate the spontaneous firing rate. This protocol was repeated two times for each session. The stimulus was time stamped and synced to the Axona recordings and the delay from the monitor $(30 \mathrm{~ms})$ was accounted for in the analysis.

\section{Monocular deprivation}

When stable cells were recorded over several sessions (1-2 d), animals in the MD groups were briefly anesthetized with isoflurane and the eyelids of 1 of the eyes were sutured shut with $4-5$ stitches. The animals were allowed to wake up and recover in their home cage for 10-15 min before they were connected to the recording equipment and spiking activity was recorded in response to visual stimulation of the nondeprived eye. This recording served as baseline activity. After $12 \mathrm{~h}$, the responses of the same neurons were again recorded and similarly after 24 and $48 \mathrm{~h}$. Beyond this point, it became difficult to confirm the identity of the neurons; these data were therefore excluded from analysis. The suture was monitored twice a day. After $5 \mathrm{~d}$, the animals were anesthetized by a subcutaneous injection of a mixture of fentanyl $(0.08 \mathrm{mg} / \mathrm{kg})$, fluanizone $(2.5 \mathrm{mg} / \mathrm{kg})$, and midazolam $(0.25 \mathrm{mg} / \mathrm{kg})$ and the suture was removed. The animals were placed on a heating pad in front of a computer monitor $(25 \mathrm{~cm}$ from eyes to monitor, covering $\sim 60-70^{\circ}$ of visual space) and visual responses recorded while alternating covering of one of the eyes to determine ocular dominance preferences. In addition to this, two animals in each group were anesthetized with the fentanyl/fluanizone/midazolam mixture before eye closure to determine baseline ocular dominance index (ODI).

\section{Histology}

At the end of the experiments, animals were deeply anesthetized by an intraperitoneal injection of pentobarbital sodium $(50 \mathrm{mg} / \mathrm{kg})$ and perfused intracardially with $0.9 \% \mathrm{NaCl}$, followed by $4 \%$ paraformaldehyde (PFA) in $1 \times$ PBS. The brains were dissected out, postfixed in $4 \%$ PFA overnight, cryoprotected in $30 \%$ sucrose in $1 \times$ PBS for $3 \mathrm{~d}$, and $40 \mu \mathrm{m}$ coronal sections spanning V1 were cut with a cryostat. Staining procedures were performed on free-floating sections under constant agitation unless mentioned otherwise. The lectin Wisteria floribunda agglutinin (WFA), which recognizes $\mathrm{N}$-acetylgalactosamine-containing epitopes in the CS-GAG chains (Härtig et al., 1992), was used to visualize PNNs. The sections were rinsed 3 times in $1 \times$ PBS and blocked with $2 \%$ goat serum with $0.3 \%$ Triton $\mathrm{X}-100$ in $1 \times \mathrm{PBS}$ for $1 \mathrm{~h}$ at room temperature (RT).
The sections were then incubated with biotin-conjugated WFA (RRID: AB_2620171, Sigma-Aldrich) in blocking solution (1:200) overnight at $4^{\circ} \mathrm{C}$. On the following day, the sections were rinsed with $0.3 \%$ Triton $\mathrm{X}-100$ in $1 \times \mathrm{PBS}$, endogenous peroxidase activity was quenched for 3 min with $2 \% \mathrm{H}_{2} \mathrm{O}_{2}$ in distilled water $\left(\mathrm{dH}_{2} \mathrm{O}\right)$, and the sections were incubated with $\mathrm{ABC}$ solution (ABC Peroxidase Standard Staining Kit; Thermo Fisher Scientific). After rinsing the sections in Tris nonsaline (TNS), staining was visualized by adding a 3,3-diamonobenzidine hydrochloride (Sigma-Aldrich) solution $(0.67 \mathrm{mg} / \mathrm{ml} 0.05 \mathrm{M}$ Tris- $\mathrm{HCl}$, with $0.8 \mu \mathrm{l}$ of $\mathrm{H}_{2} \mathrm{O}_{2} / \mathrm{ml}$ ) for 3-10 min. Sections were rinsed in TNS, mounted on coverslips, and dried for $1 \mathrm{~h}$. Sections were then coverslipped with $\mathrm{dH}_{2} \mathrm{O}$ and photographed through an Axioplan 2 microscope (Carl Zeiss). High-resolution images were then stitched together using the MosaiX extension in the AxioVision software (Carl Zeiss). The following day, the sections from animals with tetrode implants were rinsed in $\mathrm{dH}_{2} \mathrm{O}$ and counterstained for Nissl substance using cresyl violet, coverslipped with Entellan, and the tetrode track identified and photographed as described above.

In addition, a few sections were collected from the animals used for histology alone. They were rinsed in $1 \times$ PBS, blocked with $2 \%$ NGS, $0.3 \%$ Triton $\mathrm{X}-100$ in $1 \times \mathrm{PBS}$, and incubated with biotin-conjugated WFA (Sigma-Aldrich) in blocking solution $(1: 200)$ overnight at $4^{\circ} \mathrm{C}$. On the following day, they were rinsed with $1 \times$ PBS and incubated with anti-CS 6 (RRID: AB_94517, Millipore) or anti-parvalbumin (RRID: AB_2313848, Swant) in $1 \times$ PBS (1:500) overnight at RT. The next day, sections were rinsed with $1 \times$ PBS and incubated with streptavidin Alexa Fluor 488 (RRID: AB_2336881, Invitrogen) and anti-mouse IgG Alexa Fluor 594 (RRID: 2535789, Invitrogen) or anti-goat (RRID: AB_2534105, Invitrogen) in $1 \times$ PBS (1:1000 and 1:1000, respectively) for $2 \mathrm{~h}$ at RT. Sections were rinsed in $1 \times$ PBS, mounted on Superfrost slides, and coverslipped with the antifading agent FluorSave (Millipore). Images were acquired using an Axioplan 2 microscope as described above, with a fluorescence light source (HXP 120 Kubler Codix, Carl Zeiss).

\section{Quantification of PNNs}

Animals were humanely killed 3, 10, 14, 17, 21, 30, and $60 \mathrm{~d}$ after injection of chABC and sections stained for PNNs as described above. Three columns of $100 \times 800 \mu \mathrm{m}$ perpendicular to the cell layers were selected within the chABC-treated area of two sections from two animals at each time point, as well as an internal control area in each section in the primary auditory cortex, and gray scale intensity was measured using ImageJ. After $3 \mathrm{~d}$, the injected area was completely void of WFA staining; therefore, the mean ratio between the control area and the injected area in these sections $3 \mathrm{~d}$ after chABC treatment was set as a reference $(0 \%$ regeneration).

\section{Data analysis}

Brain state quantification. Brain state was quantified based on the method used by Wang et al. (2013). Briefly, the power for each $0.5 \mathrm{~s}$ segment of LFP was calculated in frequency ranges between 1 and $10 \mathrm{~Hz}$ (lowfrequency, synchronized) and $30-70 \mathrm{~Hz}$ (high-frequency, desynchronized). The segments were then clustered using fuzzy c-means clustering and a ratio between low-frequency power and high-frequency power computed for each segment. Segments in the low-frequency group were included if the low/high ratio was lower than the (mean - SD) of the cluster and segments in the high-frequency group were included if the ratio was higher than the (mean $+\mathrm{SD})$ of the cluster. The segments were then extracted and analyzed separately for spiking activity.

Single units. Spike sorting was performed using the graphical clustercutting software Tint (Axona) in a semiautomatic manner. Clustering was performed manually in $2 \mathrm{D}$ projections of the multidimensional parameter space (consisting of waveform amplitudes) in combination with the KlustaKwik algorithm. Autocorrelation and cross-correlation were used as additional tools to separate spikes and to assess the quality of separation because each cluster of a single given unit should have a clear refractory period $(>1.5 \mathrm{~ms})$. Separation quality was assessed further using the Mahalanobis distance and by visual inspection. On this basis, $\sim 30 \%$ of recorded units were left out of the analysis. 
Putative excitatory neurons were distinguished from putative inhibitory neurons based on characteristics of their mean waveform, as recorded by the electrode in which the amplitude was largest (Bruno and Simons, 2002; Barthó et al., 2004; Niell and Stryker, 2008). Narrowspiking units have short baseline-to-peak and peak-to-trough times and high amplitude of the trough compared with broad spiking units. By combining these measures, we could separate the broad-spiking (putative excitatory) and narrow-spiking (putative inhibitory) neurons.

Stimulus evoked activity was separated from spontaneous activity and divided into $50 \mathrm{~ms}$ time bins. Maximum spiking activity was defined by averaging the stimulus-evoked activity in $50 \mathrm{~ms}$ bins covering the period from 50-200 ms after stimulus. Spontaneous activity was averaged between 200 and 50 ms before stimulation onset, when the gray screen was shown.

LFP. Gamma events were detected using the method described in Colgin et al. (2009). Briefly, events of $160 \mathrm{~ms}$ duration were detected in which the gamma $(30-100 \mathrm{~Hz})$ power exceeded 2 times the SD for average gamma power. Duplicated events as a result of overlapping windows were discarded and final event windows of $400 \mathrm{~ms}$ were constructed. The signal was normalized by $z$-scoring and the power calculated.

For LFP power analysis, custom MATLAB code was used. To remove line noise, a band-stop Butterworth filter of first order with a Kaiser window was applied to the frequency range $49.8-50.2 \mathrm{~Hz}$. To compute LFP power, spontaneous and stimulus bins (or extracted gamma events) were collected and the signal averaged over all trials. Power was calculated for each frequency band by $z$-scoring. Power spectral density (PSD) was estimated by the Welch method provided by MATLAB with a window length of 1024 and a $70 \%$ overlap. Time frequency representation (TFR) was generated by convolving the LFP signal with a Morlet wavelet in the frequency domain. Filter frequencies $w$ are spread linearly and bin sizes increase linearly as a function of signal frequency, denoted as $f$ as follows:

$$
\psi=C \exp \left(\frac{-(f-w)^{2} K}{2 f}\right)
$$

where

$$
C=\sqrt{1+\exp \left(-f^{2}\right)-2 \exp \left(-\frac{3 f^{2}}{4}\right)}
$$

Here, $K$ is an arbitrary quality factory set to the value of 10 . To estimate differences in spontaneous and evoked recording sessions, the LFP was binned relative to stimulus onset. The PSD and TFR were further calculated for each bin and averaged over trials.

Computational model. To investigate possible network effects of changes in synaptic efficacy, a computational model of sparsely connected integrate-and-fire neurons was used (Brunel, 2000). This model describes the membrane potential of a neuron in terms of the synaptic inputs and the synaptic currents that it receives. The synaptic inputs to the neuron are considered to be stochastic and are described as a temporally homogeneous Poisson process. To simulate a network in visual cortex, two homogenous populations of inhibitory and excitatory neurons that were sparsely interconnected were used.

The spiking variability of the neural network was quantified by calculating the average coefficient of variation of the interspike intervals. The network was simulated $1 \mathrm{~s}$ for different values of excitatory efficacy and relative inhibition, all parameters were chosen as described in model A in Brunel, 2000. All simulations were performed with the Neural Simulation Technology Initative (NEST) simulator (Gewaltig and Diesmann, 2007).

Statistics. Statistical analyses were performed in GraphPad Prism 6 and SigmaPlot 12.0. All data were tested for normality before analysis (by Shapiro-Wilk test) and statistical testing was performed accordingly.

For population data shown in box plots, lines denote the $25^{\text {th }}, 50^{\text {th }}$, and $75^{\text {th }}$ percentile and error bars show the $10^{\text {th }}$ and $90^{\text {th }}$ percentile. The population mean is marked by a cross. In the scatter plots, the line indicates the median.

\section{Results}

Degradation of chondroitin sulfate in vivo by injection of the bacterial enzyme chABC removes PNNs efficiently (Brückner et al., 1998; Kwok et al., 2008). However, the rate at which PNNs regenerate after enzymatic degradation is largely unknown. To establish a time frame for electrophysiological recordings, we determined the time that it takes for PNNs to regenerate after chABC treatment (Fig. 1). Animals were injected with chABC in V1, killed at seven different time points, and the amount of PNNs estimated by staining with WFA. As described previously (Härtig et al., 1992), most of the PNNs in V1 overlap with PV-expressing neurons (Fig. 1A). The chABC treatment eliminated almost all WFA signal within the first time point at $3 \mathrm{~d}$ after injection and a gradual increase in WFA intensity was observed, reaching 30\% after $14 \mathrm{~d}$ and almost $90 \%$ after $60 \mathrm{~d}$ (Fig. 1D).

Having established the rate of $\mathrm{PNN}$ regeneration, we performed electrophysiological recordings 3-14 d after chABC treatment (experimental timeline is shown in Fig. 1E). All electrophysiological recordings were conducted in the V1 as verified by postmortem histochemistry to confirm electrode position (Fig. 1B). Units were classified based on properties of their average waveforms (Bruno and Simons, 2002; Barthó et al., 2004; Niell and Stryker, 2008). Two separable clusters were found, corresponding to narrow-spiking (putative inhibitory) and broadspiking (putative excitatory) neurons. Approximately 20\% of both the control and chABC-treated populations were characterized as narrow-spiking, putative inhibitory (pIN) units (Fig. $2 A$, green), matching the distribution found in the neocortex in general (Markram et al., 2004).

As the distribution of excitatory and inhibitory units was unaffected by PNN degradation, we investigated whether their activity was different. It has been suggested that the PNN may facilitate the high-frequent spiking activity of $\mathrm{PV}+$ neurons (Brückner et al., 1993; Härtig et al., 1999). Therefore, we investigated whether chABC treatment affects basic properties of unit spiking activity and, indeed, the pIN units from the chABCtreated animals (4 rats, 22 units) showed a lower mean spiking activity compared with control animals (4 rats, 20 units; Fig. 2B, $p=0.0009$, Mann-Whitney $U$ test), whereas the mean activity of putative excitatory ( $\mathrm{pEX}$ ) units was unaffected by the treatment (Fig. $2 B, p=0.054$, Mann-Whitney $U$ test). This was accompanied by changes in spiking variability, measured by the coefficient of variation of interspike interval times, which was significantly higher in chABC-treated rats both for pIN $(p=0.02)$ and pEX units $(p=0.002)$ (Fig. $2 C$, Mann-Whitney $U$ test). We also examined the effect on spontaneous activity (without visual stimulation) and stimulus-evoked activity (recorded during visual stimulation). To refine the analysis, we implemented brain state as an additional measure because recent work has shown that the activity of neurons in V1 is strongly modulated by brain state, with overall higher activity when the animal is attentive (Bereshpolova et al., 2011; Wang et al., 2014; Zhuang et al., 2014). We examined events in the LFP recordings to classify trials as synchronized LFP (nonattentive) or desynchronized LFP (attentive) before analysis of unit activity (Fig. 3A). Units were then normalized according to brain state and the data pooled (Fig. $3 B$ ). For the pIN units, the reduction in activity as a result of the chABC treatment was of the same magnitude for both brain states during spontaneous and stimulus-evoked activity (Fig. $3 B$, right). The activity of pEX units was not different between groups in either state, but there was a tendency for lower activity in units isolated from chABC-treated animals. Compared with previous findings in rodents, the responsiveness to visual stimulation was low overall (Girman et al., 1999; Niell and Stryker, 2008, 2010). This may be explained by our aim to obtain an objective measure of activity in V1 using an unbiased sampling method in which all units were 
A
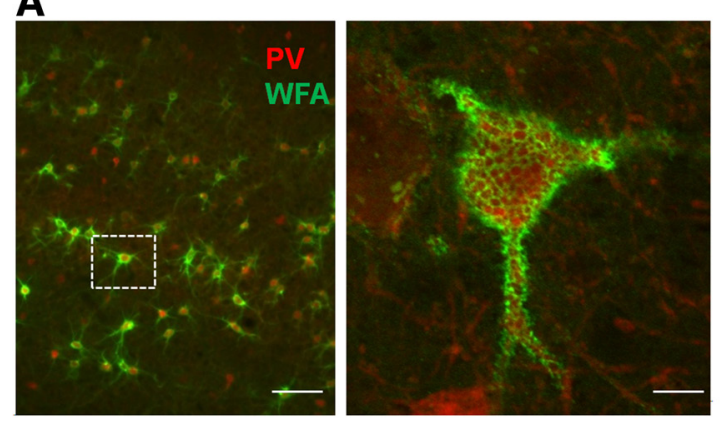

\section{B}
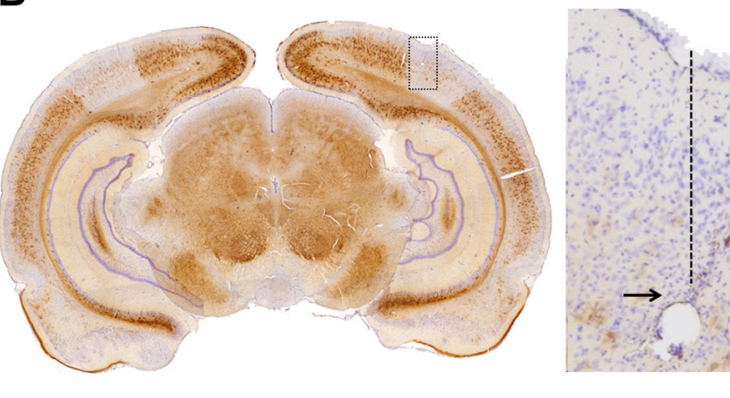

C
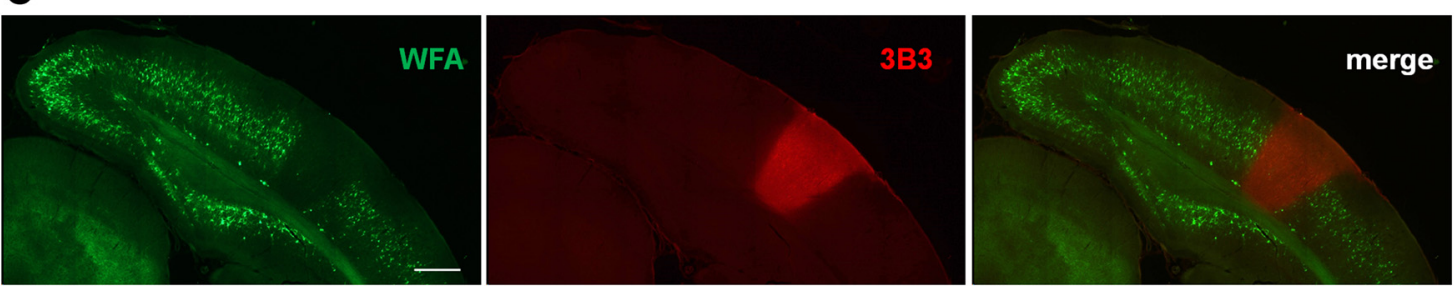

D

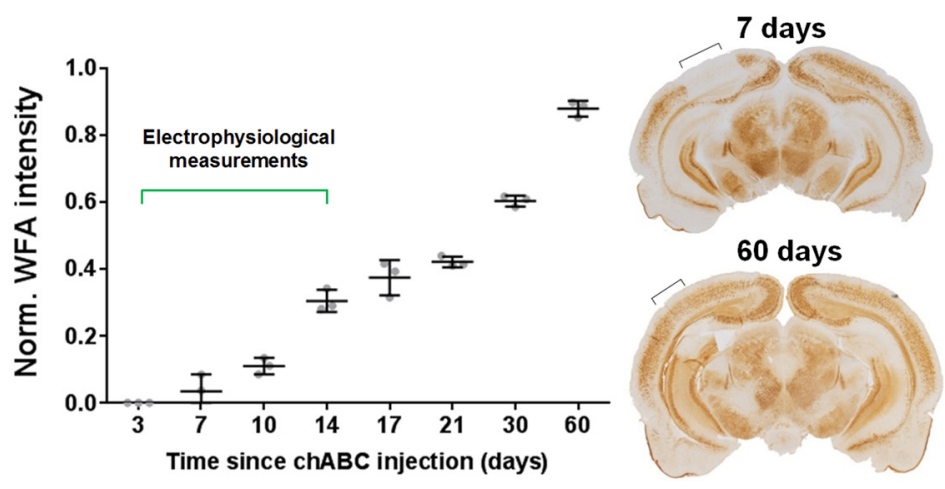

E

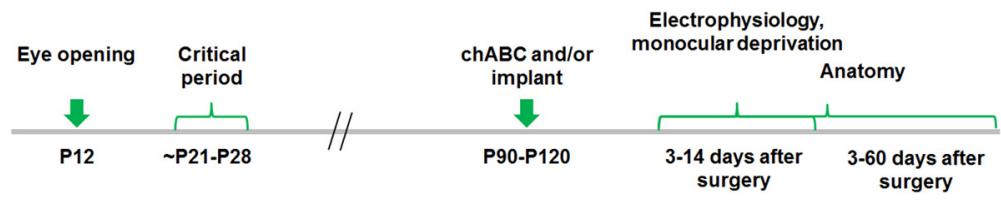

Figure 1. Histological assessment of PNNs and separation of recorded units after local injection of chABC in V1. A, WFA-positive PNNs mainly enwrap PV+ neurons in V1. Scale bars, $25 \mu \mathrm{m}$ (low magnification) and $10 \mu \mathrm{m}$ (high magnification). $\boldsymbol{B}$, chABC-treated area and the trace (dotted line) from the implanted electrodes were identified by staining with WFA in combination with cresyl violet (Nissl). Arrow indicates the end of the electrode trace at $\sim 700 \mu \mathrm{m}$ into the cortex. C, D, Regeneration of PNNs after chABC treatment was quantified in sections stained with WFA and anti-chondroitin sulfate 6 "stub" (3B3 epitope) to identify the treated area. Scale bar, $1 \mathrm{~mm}$. Animals were killed at different time points after chABC injection and WFA intensity was quantified using ImageJ. Two animals were used for each time point and intensity measurements were conducted for two sections from each animal and normalized to a reference area in the primary auditory cortex. $\boldsymbol{E}$, Timeline of the experimental scheme.

included with no selection for each unit's preferred visual stimulation. Moreover, in these awake recordings, the animals moved relative to the monitors where the stimulation was presented, thereby varying the rat's visual field. Furthermore, recent work has shown that the visual field in freely moving rats is mostly above the rat's head (Wallace et al., 2013).

Having observed the alterations in spiking variability and inhibitory activity as a result of the chABC treatment, we next investigated whether the effects could be reflected in changes in network activity by analyzing the LFP of the recordings. Although spiking activity reflects the output from smaller sets of active neurons, the LFP reflects the input currents to neurons in the area. The high-frequent gamma oscillations $(30-80 \mathrm{~Hz})$ are likely due to highly synchronized activity in the PV + cells (Cardin et al., 2009) and stimulus-evoked oscillations around $40 \mathrm{~Hz}$ are pronounced in anesthetized animals (Sohal et al., 2009). Gamma oscillations have also been shown to be prevalent in awake, head-fixed mice (Niell and Stryker, 2010). In comparison, in our recordings from freely moving rats, gamma oscillations were very sparse and occurred only as highly nonstationary bursts of activity across several frequencies despite a simultaneous strong visual response observed in the LFP (Fig. $4 A$, left). Therefore, to analyze this component of the LFP, we extracted events in which gamma oscillations occurred (as described in Colgin et al., 2009). Analyzing these events, we found no differences between groups either in spectral density power or number of events per 
A

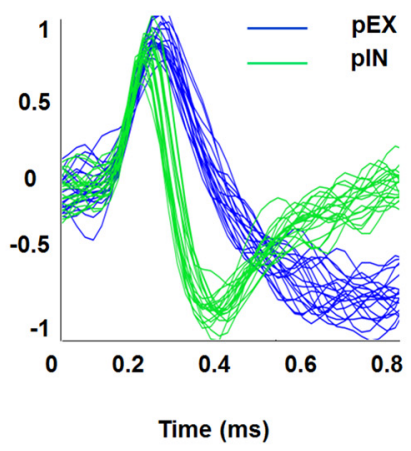

control

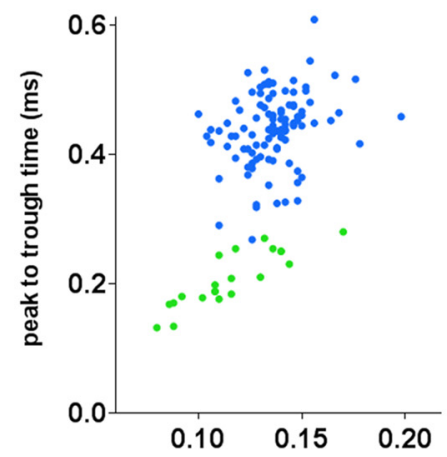

B plnhibitory

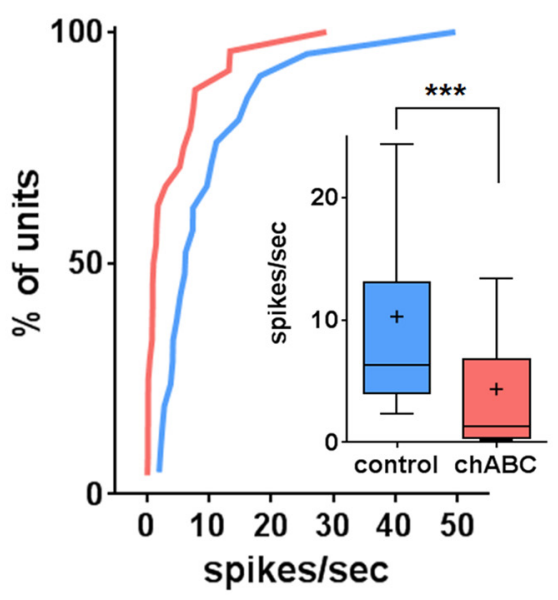

$\operatorname{chABC}$ spike half-width (ms)

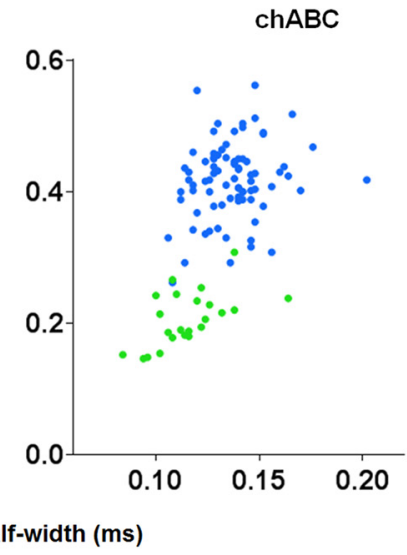

pExcitatory

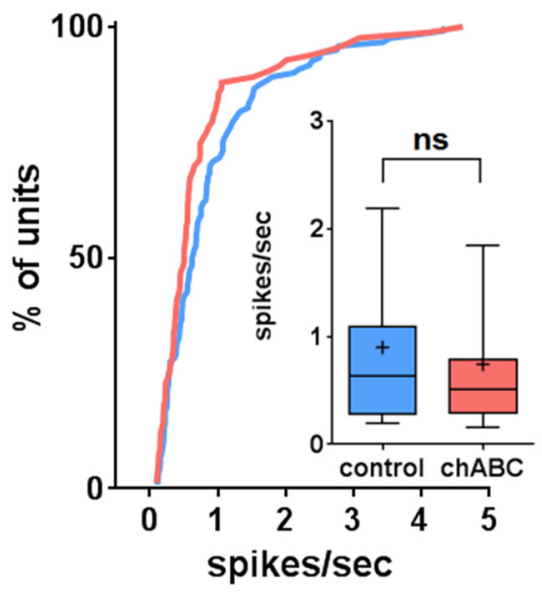

C
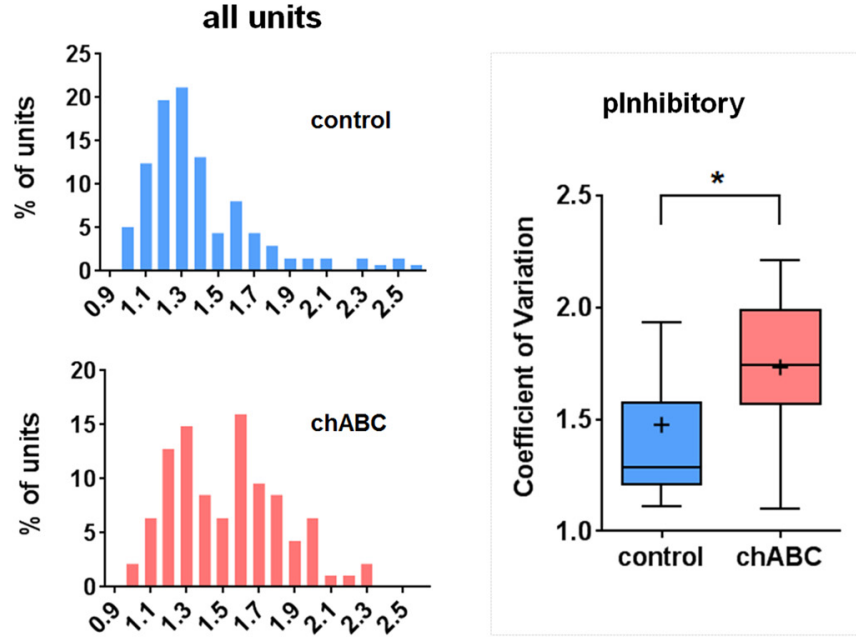

pExcitatory

\section{Coefficient of Variation}

Figure 2. chABC treatment causes reduced inhibitory activity and increased spiking variability. $A$, Isolated units were classified as excitatory or inhibitory based on characteristics of their waveform. Example traces (20 spikes) from two units, one classified as putative inhibitory (green) and one as putative excitatory (blue). The ratio in amplitude from peak to baseline and baseline to trough, half-amplitude width, and peak to trough time, were used to classify units from control animals (left) and animals treated with chABC (right). In total, $\sim 20 \%$ of the units in both groups were classified as putative inhibitory (17\%, 20 of $122 ; 22 \%, 22$ out of 105 units from control and chABC animals, respectively). $\boldsymbol{B}$, Mean spiking activity of all isolated units. Putative inhibitory units recorded from chABC-treated rats had lower mean spiking activity than controls (left, $p=0.0009, n=20$ units for control, $n=22$ units for chABC). Putative excitatory units were not significantly different between groups (right, $p=0.054, n=102$ for control, $n=83$ for chABC). Data were analyzed by Mann-Whitney $U$ test. C, Spiking variability, measured by the coefficient of variation, was higher for units from chABC-treated animals compared with controls. Panels to the right indicate significant differences for putative inhibitory units $(p=0.02)$ and putative excitatory units $(p=0.002)$ between groups. Data were analyzed by Mann-Whitney $U$ test. 
A

synchronized

de-synchronized

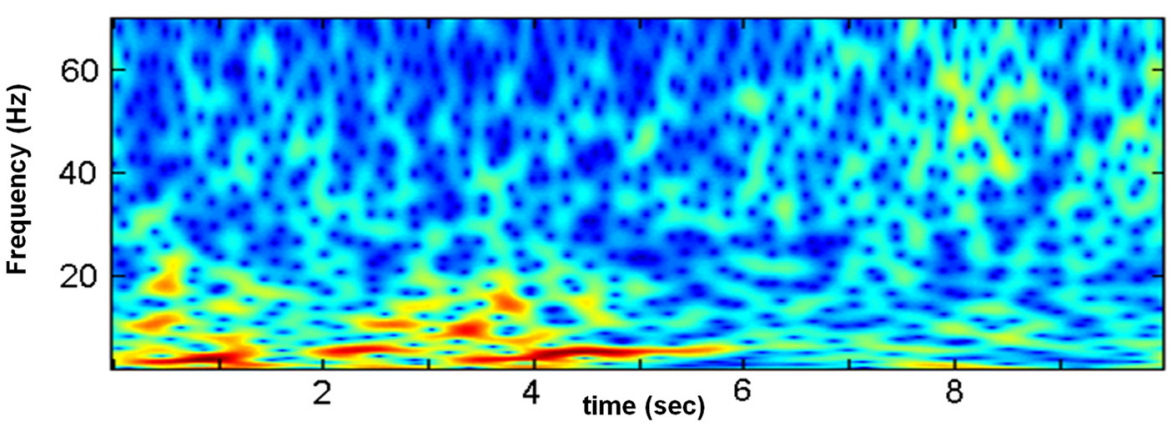

要

B

plnhibitory

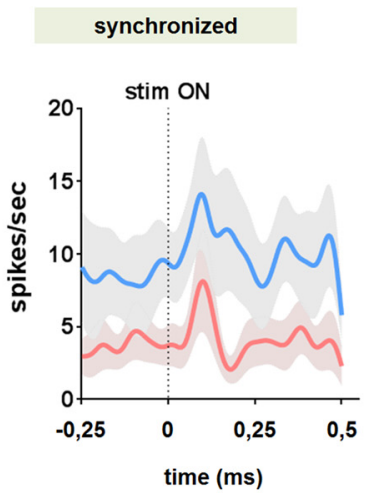

de-synchronized
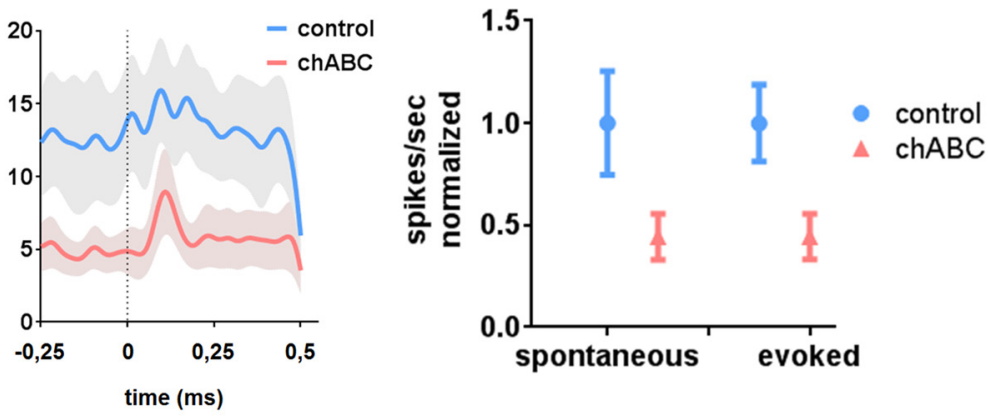

pExcitatory
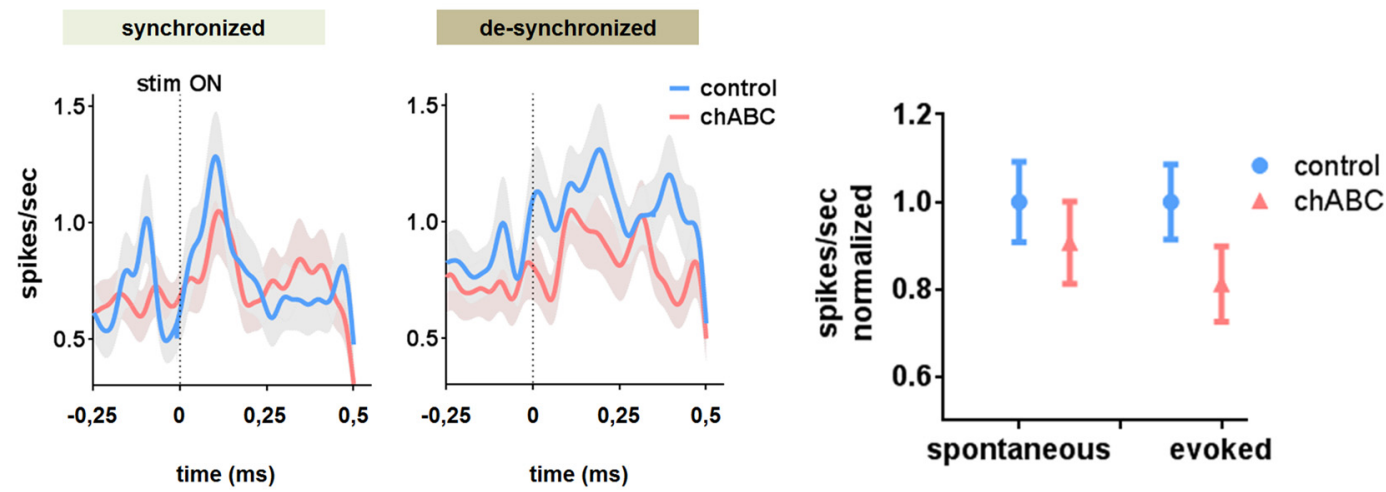

Figure 3. Population activity varies with brain state. $A$, Spontaneous switch of brain state from synchronized (nonattentive) to desynchronized (attentive) state. Top, Spectrogram computed from the LFP (wavelet analysis, log-power color-coded). Bottom, Corresponding raw LFP trace (the arrow indicates a state transition after approximately six seconds). $\boldsymbol{B}$, Brain state was classified as synchronized or desynchronized based on the ratio between the LFP power of slow $(1-10 \mathrm{~Hz})$ and fast $(30-80 \mathrm{~Hz})$ oscillations. Unit activity is shown before and during visual stimulation of all putative inhibitory (top) and excitatory units (bottom) in both brain states. Spontaneous and stimulus-evoked activity in putative inhibitory and excitatory units is shown in right panels. Inhibitory activity was consistently lower in chABC-treated rats compared with controls across brain states and stimulus-evoked/spontaneous activity. Data from both groups were normalized to the mean of the control group in each respective brain state and pooled. Data are shown as mean \pm SEM. 
A
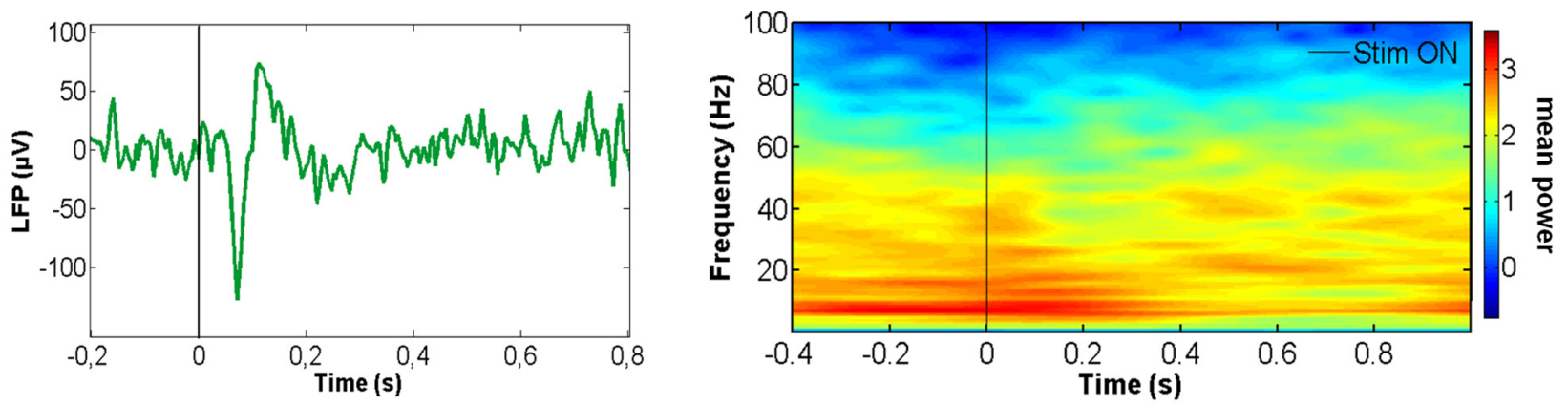

B
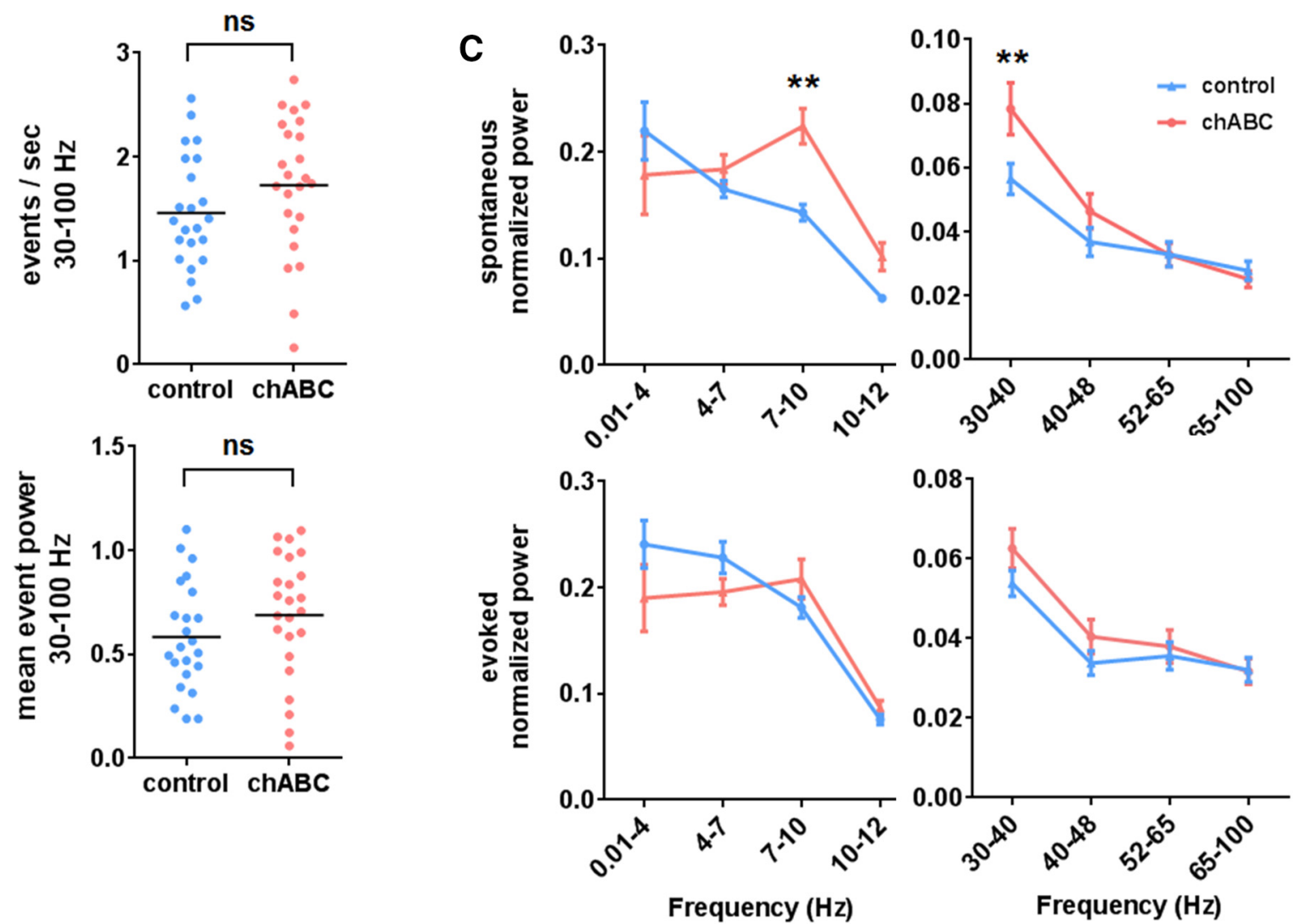

Figure 4. Removal of the PNNs affect LFP oscillations during spontaneous activity, but not during stimulus-evoked activity. $A$, Example of LFP recording (left) from one session aligned with stimulus onset $(0 \mathrm{~s}$ ) and the respective frequency spectrogram of the same recording (right). $\boldsymbol{B}$, Time periods with gamma oscillations (gamma events) were not different in chABC-treated animals, neither events per unit time nor gamma event power. $C$, During spontaneous activity, the chABC-treated rats showed significantly stronger oscillations compared with controls, both in the theta $(7-10 \mathrm{~Hz}$ ) and low-gamma $(30-40 \mathrm{~Hz}$ ) range of the LFP ( $p=0.002$ for $7-10 \mathrm{~Hz}$ and $p=0.001$ for $30-40 \mathrm{~Hz} ; n=23$ sessions from 3 rats in each group). During visual stimulation, however, we found no differences between groups. Data are shown as mean \pm SEM and were analyzed by Student's $t$ test.

unit time (Fig. 4B). This also held true when we analyzed gamma events in narrow frequency ranges (data not shown). However, when the spontaneous and stimulus-evoked periods were analyzed separately, we found stronger LFP power in chABC-treated animals both in the theta $(7-10 \mathrm{~Hz})$ and low-gamma $(30-40 \mathrm{~Hz})$ ranges during periods of spontaneous activity $(400 \mathrm{~ms}$ before and until stimulus onset; Fig. 4C, 7-10 Hz: $p=0.002$, and 30-40 Hz: $p=0.001$, Student's $t$ test). During visually evoked activity, we found no differences between groups. Therefore, the LFP recordings were consistent with the single unit responses in that the chABC treatment changed the baseline state of the neuronal network, but not the processing of visual stimuli.

To explore potential mechanisms underlying the network alterations observed, we performed a series of network simulations. To test whether changes in spiking variability could be explained by changes in the excitatory-inhibitory balance, we tested the effect of decreased inhibitory synaptic efficacy on a range of excitatory synaptic efficacies in a randomly connected balanced network of leaky integrate-and-fire neurons (Brunel, 2000). This model has become established as a canonical model for the description of spontaneous cortical activity because it captures many essential features observed in experiments; for example, asynchronous irregular spiking activity. We adjusted inhibitory synaptic efficacy for different values of excitatory synaptic efficacy and simulated activity in a network of 10,000 neurons for each adjustment with the NEST simulator (Gewaltig and Diesmann, 2007). We found that spiking variability increases as inhibitory efficacy decreases (Fig. 5). The changes in variability were true for networks of both excitatory and inhibitory neurons (Fig. 5, left and right, respectively). This state shift of the network 
A

Excitatory population

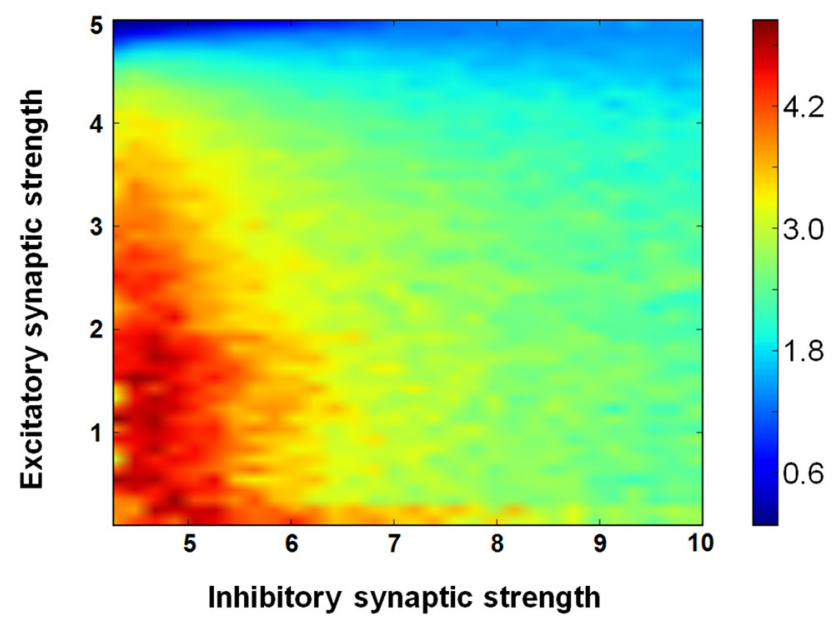

B

Inhibitory population

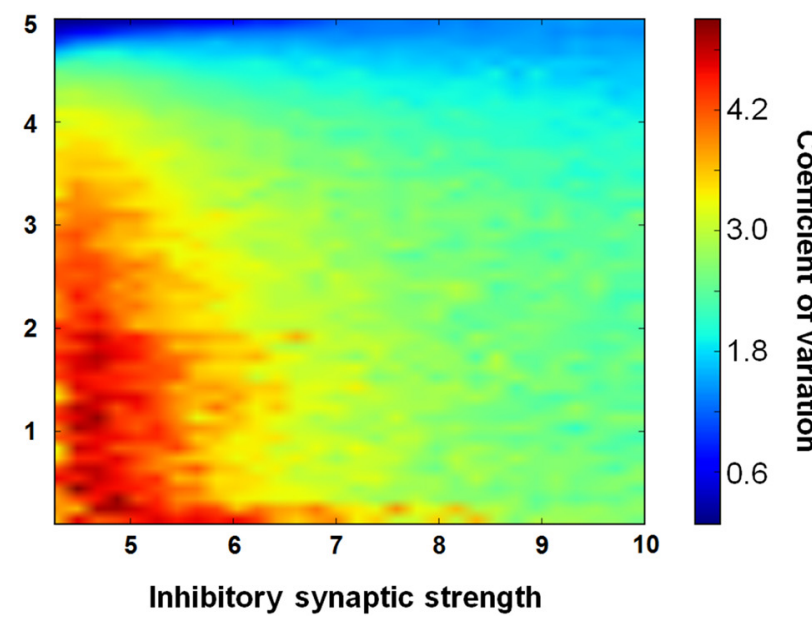

Figure 5. Reduced inhibition induces a change in the network state toward an irregular state. The effect of altered excitatory-inhibitory balance was tested in networks with 10,000 randomly connected neurons with the NEST simulator. Inhibitory synaptic efficacy was adjusted for different values of excitatory synaptic efficacy and activity simulated for each adjustment simulated. Coefficient of variation is color coded as a function of relative inhibition on the $x$-axis and excitatory synaptic strength on the $y$-axis. Separate simulations were run for each change in inhibitory and excitatory synaptic strength (each pixel represents a single simulation). Results from the excitatory and inhibitory population are depicted in $\boldsymbol{A}$ and $\boldsymbol{B}$, respectively. In both populations, the coefficient of variation increases with reduced inhibitory strength.

toward a more irregular state is consistent with our in vivo findings and supports that chABC treatment may cause a shift in the excitatory-inhibitory balance, correlating with changes in spiking variability and spiking activity.

Alteration of the inhibitory tone may be one of the mechanisms by which the PNNs contribute to regulate plasticity. To address this, we monitored chABC-induced OD plasticity in the adult rat cortex in the first hours after MD and after $5 \mathrm{~d}$ of MD. Although OD plasticity is a model that has been used widely to study activity-dependent plasticity, the dynamics of single units or population activity across time has not been investigated thoroughly. By recording the activity from the same units in awake animals at several time points during the first $48 \mathrm{~h}$ of $\mathrm{MD}$, we observed how single units responded to sensory deprivation over time. The animal was anesthetized with isoflurane when the eye was sutured shut and, 10-15 min later, the first awake recording was performed (experimental timeline is outlined in Fig. 6A). Units were mainly recorded from cell layers $2 / 3$ because these layers have been shown to have the most pronounced effects early during MD (Medini, 2011). In control animals that did not undergo $\mathrm{MD}$, the units' response patterns and activity levels were highly stable across several sessions (Fig. $6 \mathrm{~B}$, left). In controls that underwent $\mathrm{MD}$, units recorded in the contralateral hemisphere to the closed eye showed a nonsignificant potentiation of activity from immediately after MD start to $12 \mathrm{~h}$ later $(p=0.06$, MannWhitney $U$ test). The median firing rate returned to baseline at $48 \mathrm{~h}$. In animals treated with chABC, however, the units' activity in the contralateral hemisphere was significantly reduced after $12 \mathrm{~h}$ of MD (Fig. $6 B$, middle right, $p=0.005$, Mann-Whitney $U$ test). On a population level, these units remained in a low activity state, but some showed a rebound in activity back to baseline levels or even higher. The ipsilateral unit activity showed no change at $12 \mathrm{~h}$ after MD start, but showed a significant increase in activity after $24 \mathrm{~h}$ (Fig. $6 B$, far right, $p=0.03$, Mann-Whitney $U$ test). Therefore, our data show that MD after PNN removal first induces a shift in activity in the contralateral hemisphere and later in the ipsilateral hemisphere. This is consistent with previ- ous work performed in CP aged animals (Frenkel and Bear, 2004; Gandhi et al., 2008).

To determine whether OD plasticity occurred in the chABCtreated animals, the eye was opened after $5 \mathrm{~d}$ of $\mathrm{MD}$ and recordings conducted under anesthesia. To assess the effect of MD, an ODI was computed for each unit in response to monocular stimulation as follows: ODI $=(C-I) /(C+I)$ where $C$ and $I$ represent, respectively, the mean stimulus-evoked firing rate from stimulating the contralateral or ipsilateral eye (the index ranges from -1 for neurons driven exclusively by the ipsilateral eye to +1 for those driven exclusively by the contralateral eye). We found no effect on ODI in adult control rats, whereas the chABCtreated rats showed a significant shift in ODI in favor of the open (ipsilateral) eye (Fig. 6C; untreated control vs chABC MD $p=$ 0.0002 , MD control vs MD chABC $p=0.001$, Student's $t$ test). These findings are consistent with previous work (Pizzorusso et al., 2002).

Activity-dependent plasticity is likely to work at the network level, so we examined the population responses in the LFP recordings during MD. Although events of gamma oscillations were sparse in both controls and chABC-treated animals in baseline conditions, $\mathrm{MD}$ in chABC-treated rats induced abrupt and strong oscillations $\sim 40$ and $55 \mathrm{~Hz}$ shortly after the onset of $\mathrm{MD}$ (Fig. 7 $A, B$ ). These high-frequency oscillations were abundant in all chABC-treated rats that underwent $\mathrm{MD}$, but disappeared gradually $\sim 1 \mathrm{~h}$ after the eye closure $(52-65 \mathrm{~Hz}$, repeatedmeasures ANOVA, $F=8.69, p=0.01$ and $35-48 \mathrm{~Hz}, F=10.13$, $p=0.01)$. The LFP effects were observed both in the ipsilateral and contralateral hemispheres to the closed eye, although they were strongest in the contralateral side. The gamma activity was highly synchronized between the two hemispheres (Fig. 7C). In contrast, control animals that underwent MD displayed no increase in gamma oscillations. This novel observation suggests that an immediate increase in the synchrony of PV + activity occurs after $\mathrm{MD}$, but only in a network with high plasticity.

The single unit and population responses to MD in chABCtreated animals suggest that removal of PNNs resets the neuronal 
A

\begin{tabular}{ccccc}
$\begin{array}{c}\text { Eye } \\
\text { opening }\end{array}$ & $\begin{array}{c}\text { Critical } \\
\text { period }\end{array}$ & $\begin{array}{c}\text { chABC and/or } \\
\text { implant }\end{array}$ & $\begin{array}{c}\text { Eye closure, activity } \\
\text { recorded every 12 } \mathrm{h} \\
\text { (awake) }\end{array}$ & $\begin{array}{c}\text { Eye opening, ODI } \\
\text { measurement } \\
\text { (anesthesia) }\end{array}$ \\
\hline $\mathrm{P} 12$ & $\sim \mathrm{P} 21-\mathrm{P} 28$
\end{tabular}

B
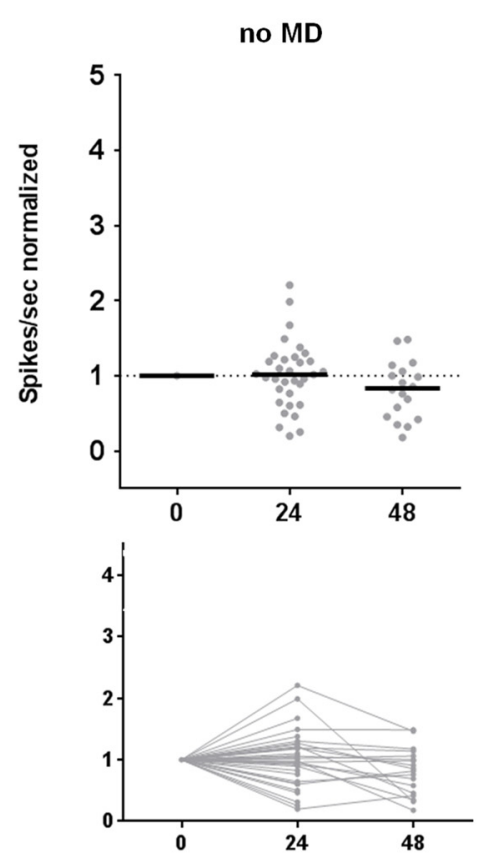

Time since MD start (hours)

C

\section{controls}
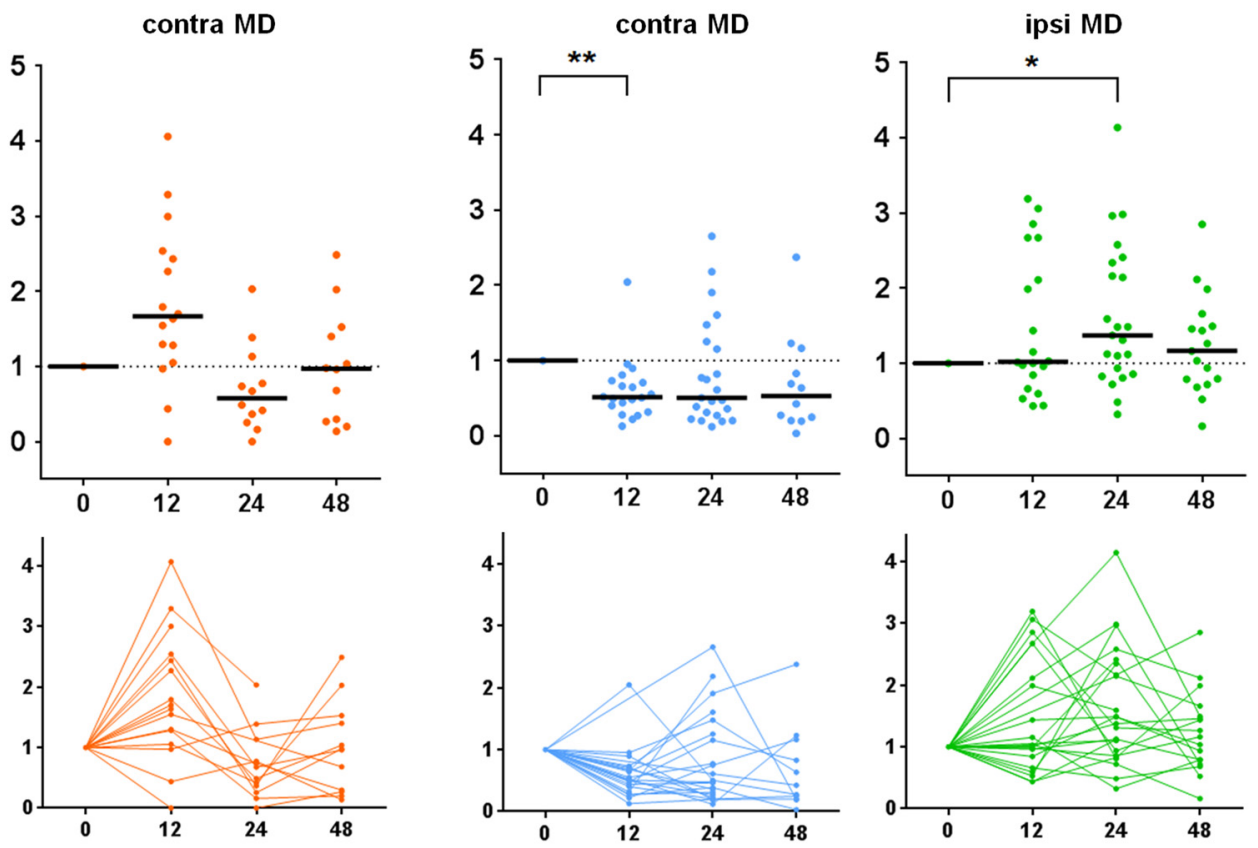
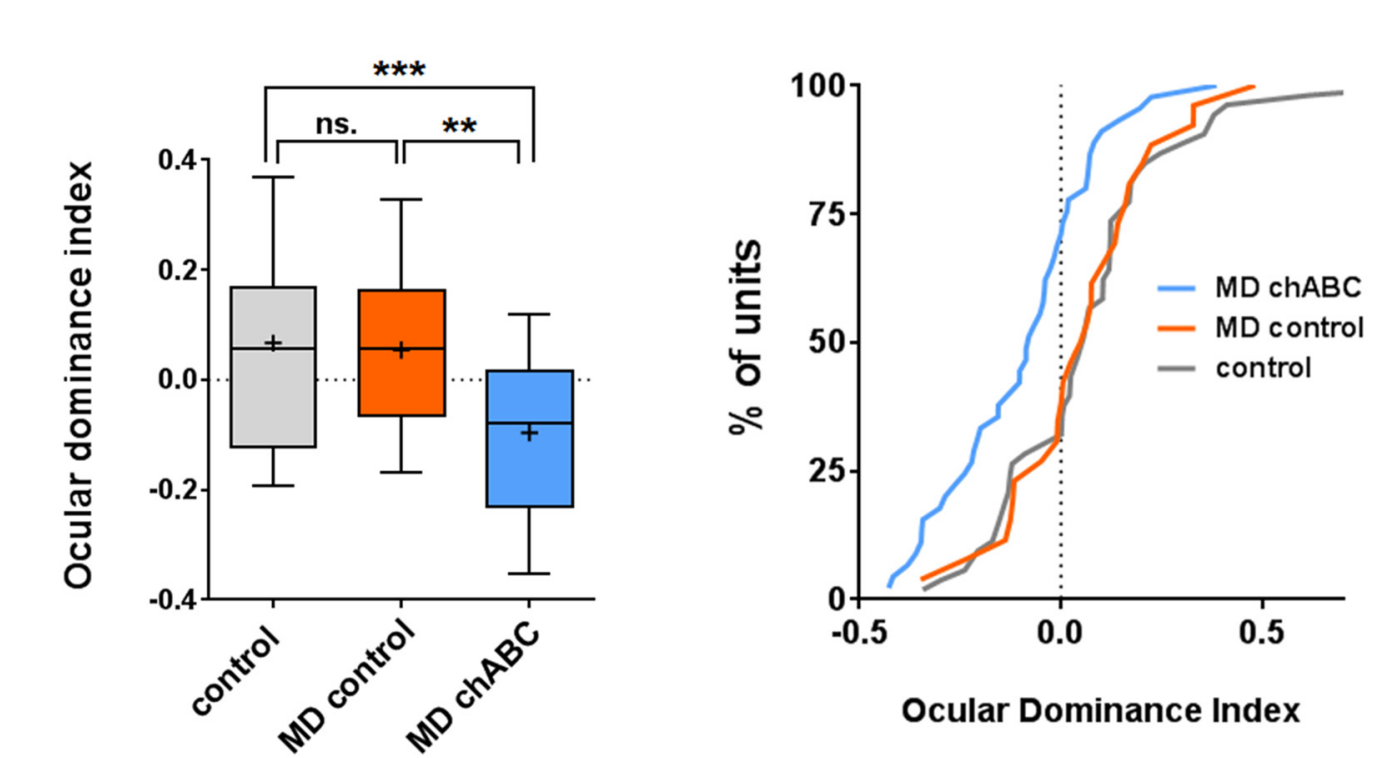

Figure 6. Heightened OD plasticity after enzymatic degradation of PNNs. A, Timeline of the experiment with removal of PNNs in adult animals followed by MD and electrophysiological recordings. $B$, Chronic recordings of single units during the first $48 \mathrm{~h}$ of MD. Data were normalized to the first recording $\left(t_{0}\right)$ made $\sim 15 \mathrm{~min}$ after MD start by as follows: firing rate $t_{x} /$ firing rate $\left.t_{0}\right)$. Top shows population activity; thick line indicates population median. Bottom shows the single units' responses over time. In control animals, activity of the units was stable over time. In control animals after $M D$, there was a nonsignificant potentiation of activity after $12 \mathrm{~h}(p=0.06)$, which went back to baseline activity after $48 \mathrm{~h}$. In chABC-treated animals, units contralateral to the deprived eye showed a significant reduction in activity after $12 \mathrm{~h}(p=0.005)$, whereas units ipsilateral to the deprived eye were potentiated after $24 \mathrm{~h}(p=0.03)$. Data were analyzed by Mann-Whitney $U$ test. $C$, After $5 \mathrm{~d}$ of MD, responses to visual stimulation shifted toward the ipsilateral (open) eye in chABC-treated animals, but not in control animals. ODI was computed from the visually evoked responses to monocular stimulation, $0 \mathrm{DI}=$ (contralateral - ipsilateral)/(contralateral + ipsilateral). $\mathrm{MD}$ chABC: 45 units ( 4 animals), control: 53 units ( 3 animals), $M D$ control: 2 animals ( 32 units), $p=0.0002$ (control vs chABC MD), $p=0.001$ (control MD vs chABC MD), $p=0.8$ (control vs control MD). Data were analyzed by Student's $t$ test. 
A

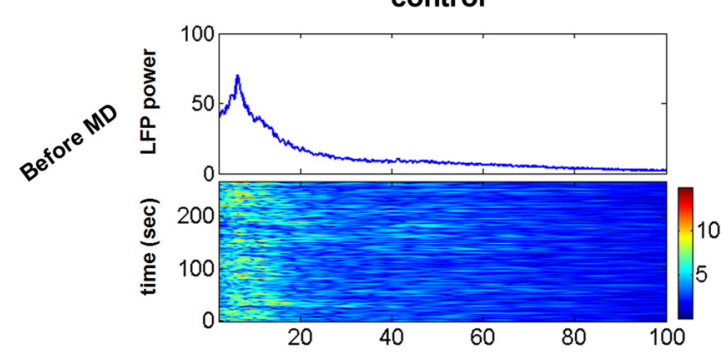<smiles>CCCCCC</smiles>

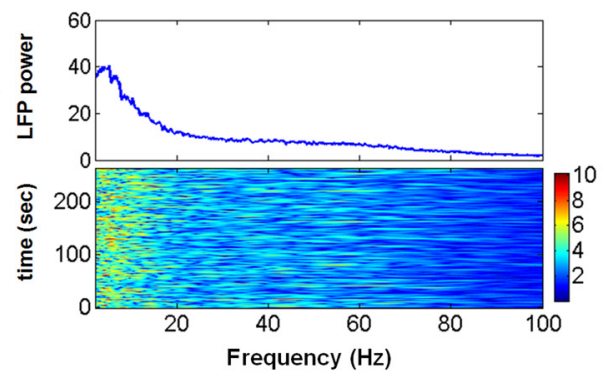

chABC
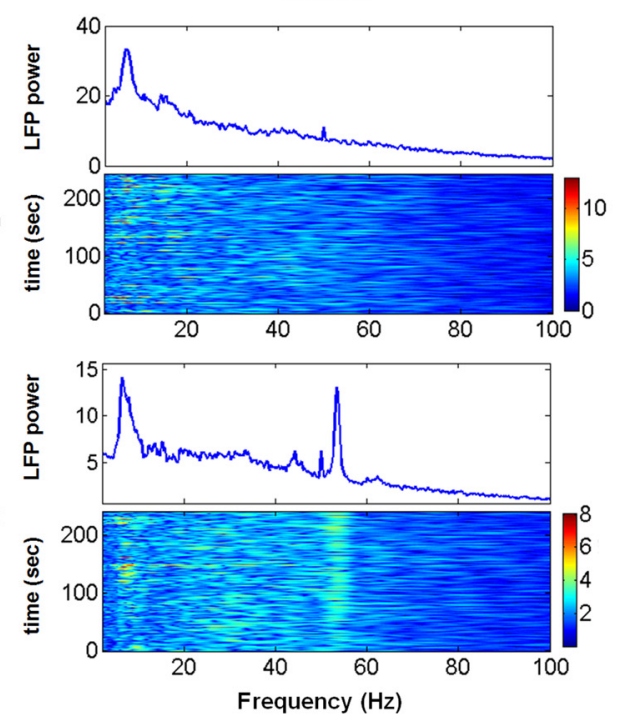

B control
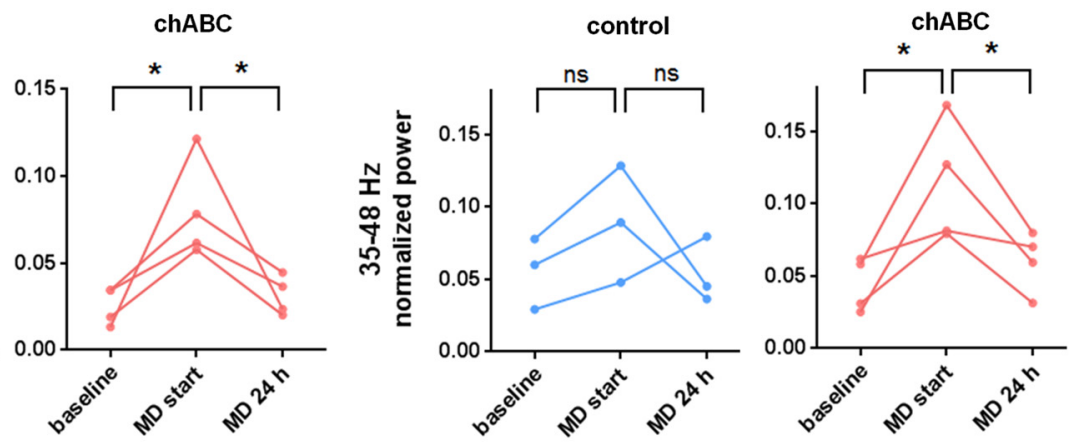

C

MD start in chABC treated animal
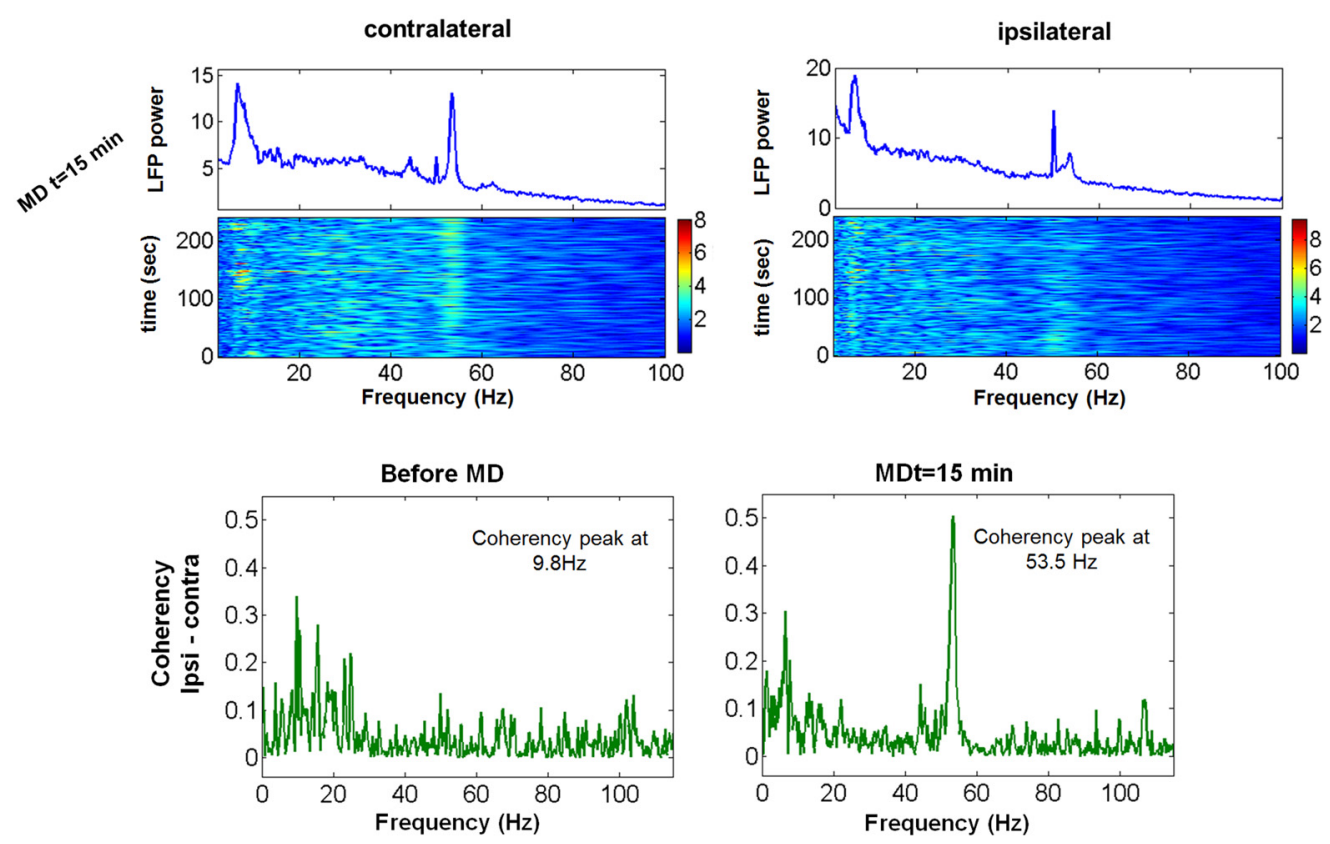

Figure 7. MD induces gamma oscillations after PNN removal of adult rats, but not in controls. $A$, Example recordings from a control animal and one treated with chABC just before and immediately after the start of MD. Data are shown as a power spectrum and a color-coded wavelet of LFP power. The MD in chABC-treated animals caused gamma activity at $\sim 40$ and $55 \mathrm{~Hz}$ in the LFP (peak at $54 \mathrm{~Hz}$ ). The small peak at $50 \mathrm{~Hz}$ reflects local line noise and is not biological activity (filtered out for power analysis). $\boldsymbol{B}$, Left, Repeated-measures ANOVA revealed no differences between 52 and 65 $\mathrm{Hz}$ in control animals ( 3 rats, $F=0.85, p=0.5$ ), but a significant effect in chABC-treated animals ( 4 rats, $F=8.69, p=0.01$ ). Tukey's multiple-comparisons (Figure legend continues.) 
A

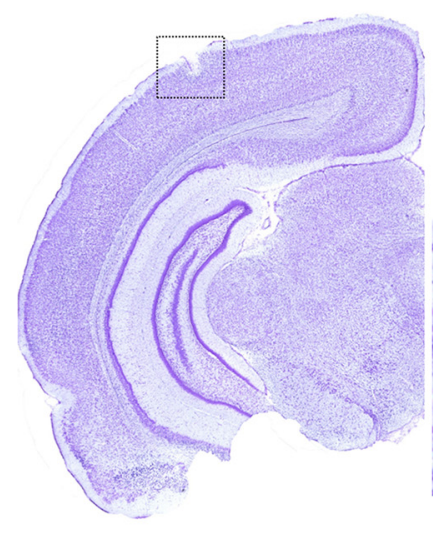

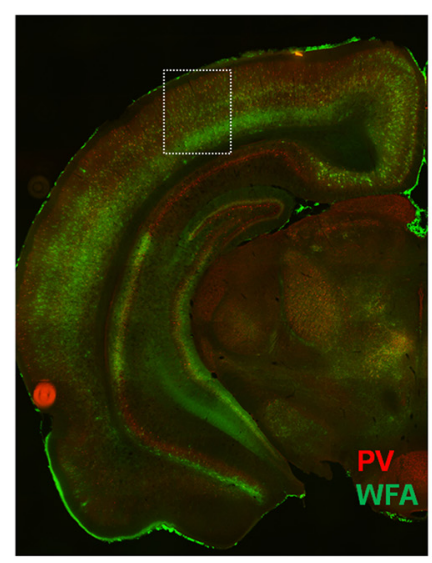

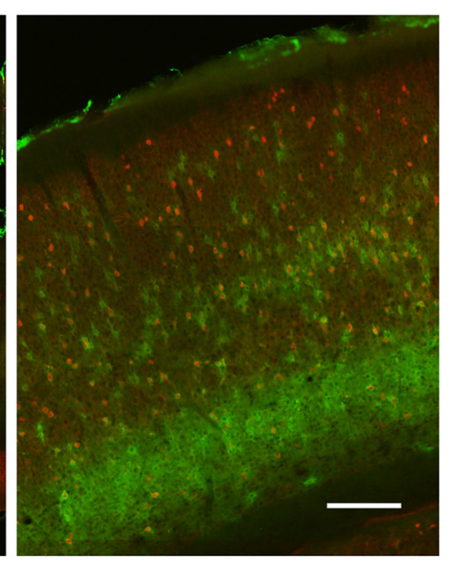

B

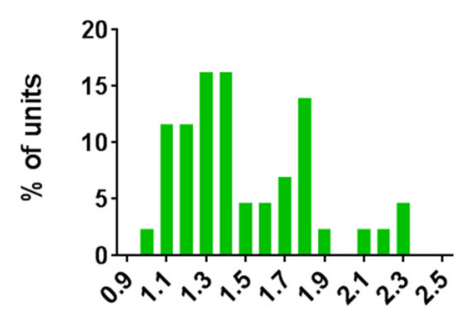

Coefficient of Variation

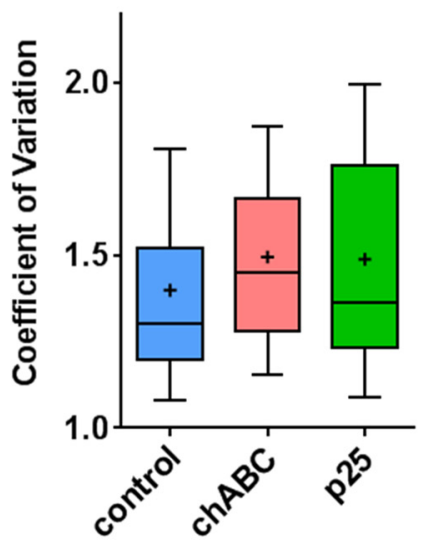

\section{C}
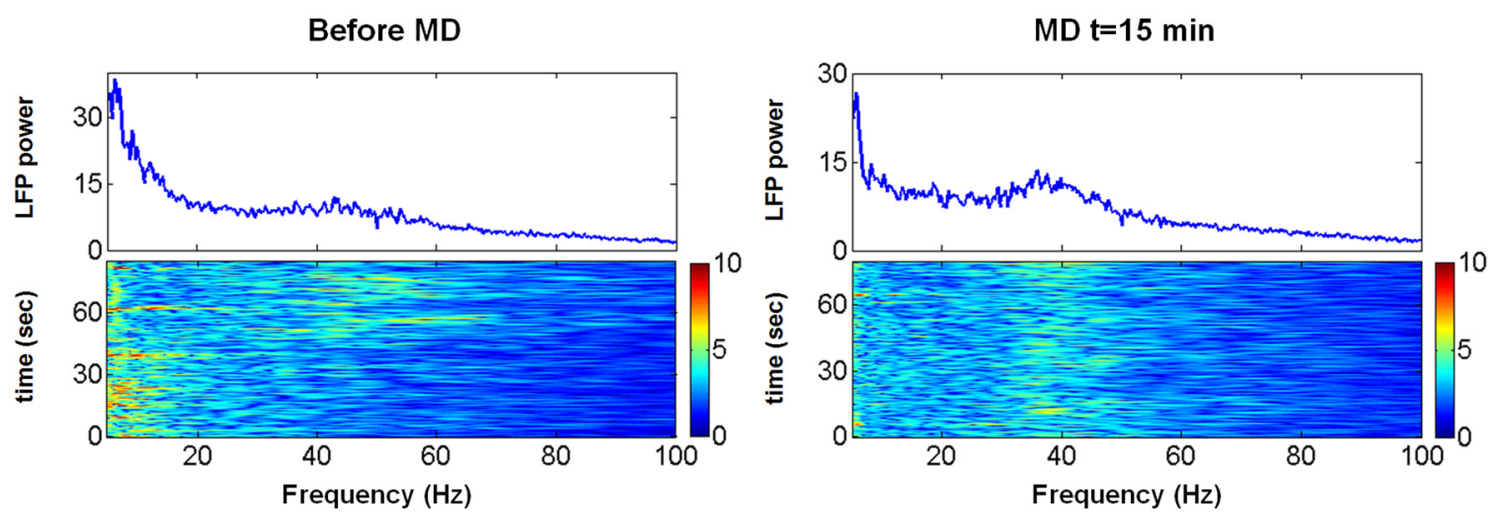

Figure 8. Electrophysiological recordings from V1 ofjuvenile rats. $A$, Nissl-stained section indicating the trace (dotted line) of the electrodes in V1 (left) and a section stained for PNNs (WFA, green) and PV (red) from a rat killed at p27. Scale bars, $100 \mu \mathrm{m}$. B. Coefficient of variation of unit spiking activity was similar between juvenile and adult chABC-treated animals ( $p=0.68$, chABC vs p25, Mann-Whitney U test). C, Example LFP recordings in a juvenile rat (p25). Data are shown as a power spectrum and a color-coded wavelet of LFP power. Note the increase in gamma oscillations after MD.

(Figure legend continued.) test revealed that the "MD start" recording was significantly different from both before MD and "MD 24 " (baseline: MD start, $p=0.02$, MD start:MD $24 \mathrm{~h}, p=0.03$, baseline: MD $24 \mathrm{~h}, p=0.9$ ). Right: Repeated-measures ANOVA revealed no differences between 35 and $48 \mathrm{~Hz}$ in control animals ( 3 rats, $F=0.45, p=0.4$ ), but a significant effect in chABC-treated animals ( 4 rats, $F=10.13, p=0.01$ ). Tukey's multiple-comparisons test revealed that the MD start recording was significantly different from both before MD and MD $24 \mathrm{~h}$ (baseline: MD start, $p=0.01$, MD start: MD 24 h, $p=0.03$, baseline: MD 24 h, $p=0.9$ ). C, Example recordings made simultaneously from the ipsilateral and contralateral hemispheres of chABC-treated rats. The gamma activity in the LFP induced by MD was highly synchronized between the ipsilateral and contralateral hemispheres, as indicated by a large increase in coherency in the LFP. network to an immature state, similar to how the network functions in juvenile animals, but this remains to be demonstrated. There is a very limited amount of data from electrophysiological recordings from the V1 in awake and freely moving juvenile animals. Therefore, to get comparable data, we recorded from three $\mathrm{CP}$ aged rats (P24-P26) in which the inhibitory network is immature and PNNs are not fully developed (Fig. 8A). We performed recordings of unit and population activity in a similar manner to that of adult rats; that is, during visual stimulation and immediately after MD. The units from the CP aged rats $(n=43$ units from 3 animals) showed similar variability in spiking activ- 
ity to that of units from the chABC-treated rats (Fig. $8 B, p=0.68$, chABC vs $\mathrm{p} 25 ; p=0.08$, control vs $\mathrm{p} 25$, Mann-Whitney $U$ test). Compared with our in vivo data from adult rats and the in silico data from the Brunel model (Fig. 5), this points to similar network states between the CP aged animals and the chABC-treated group, in which reduced inhibitory activity may cause increased spiking variability.

To compare network mechanisms directly, we also performed $\mathrm{MD}$ in the juvenile rats and analyzed the LFP recorded before and immediately after eye closure. During baseline recordings, the LFP was dominated by lower frequencies, but had short bursts of high-frequency oscillations, similar to the recordings from adults. However, upon MD start, we found a potentiation of high-frequency oscillations at $\sim 40 \mathrm{~Hz}$ (Fig. $8 C$ ), similar to what we observed in chABC-treated adult rats. This further supports that the network state in adult rats after PNN removal may be similar to the network function of CP aged rats. Moreover, it suggests that increased gamma activity shortly after the onset of sensory deprivation is a common feature of neural networks in a state of high plasticity.

\section{Discussion}

By conducting chronic extracellular recordings from V1 of adult rats, we show that enzymatic degradation of PNNs alters the activity of inhibitory neurons, increases plasticity, and causes a remarkable increase in gamma activity within minutes after onset of monocular deprivation. Plasticity at single units appears in the form of reduced activity in contralateral responsive units $12 \mathrm{~h}$ after onset of $\mathrm{MD}$, followed by a potentiation of activity of ipsilateral responsive units after $24 \mathrm{~h}$.

To date, visual responses of neurons in visual cortex in awake and freely moving rodents have rarely been examined (Ji and Wilson, 2007; Toyoizumi et al., 2013; Haggerty and Ji, 2015). The use of chronically implanted tetrodes enabled recordings in awake rats without movement restraint and monitoring the activity of the very same neurons across several sessions, bringing novel insight into the mechanisms of OD plasticity.

Our data suggest that removal of PNNs leads to reduced inhibitory activity, with only minor effects on basic information processing by the network. The latter is consistent with previous work demonstrating that PNN removal had little or no effect on response properties of excitatory neurons such as orientation tuning and visual acuity (Pizzorusso et al., 2002).

The enzyme chABC is used widely to degrade PNNs and to investigate how PNNs shape cortical processing and plasticity. Although chABC is not selective to matrix components in PNNs, global knockout of the link protein Ctrl-1, an essential component of PNNs, demonstrated that the PNN is the part of extracellular matrix responsible for plasticity regulation (Carulli et al., 2010).

Maturation of the inhibitory network, in particular the PV+ inhibitory neurons, is likely the main contributor to the reduction in plasticity during adolescence (for review, see Hensch, 2005; Levelt and Hübener, 2012). The high activity of mature $\mathrm{PV}+$ neurons may be facilitated by the PNNs by providing a favorable extracellular environment (Brückner et al., 1993; Härtig et al., 1999; Morawski et al., 2015), protection against oxidative stress after the high metabolic demands (Cabungcal et al., 2013), and capture of Otx2 (Beurdeley et al., 2012). However, evidence to support this facilitation has largely been absent. Here, we show for the first time that removal of PNNs causes reduced activity of putative inhibitory neurons (Fig. 2) during both spontaneous and visually evoked activity and across brain states
(Fig. 3), accompanied by increased spiking variability. A similar trend was observed in juvenile rats, in which inhibition is lower than in adults, suggesting a common feature of an immature network state (Hensch et al., 1998). These findings were also supported by the simulation in which the relationship between variability and inhibition was investigated. Adjusting inhibitory synaptic efficacy alone was sufficient to cause increased spiking variability (Fig. 5), supporting the hypothesis that the level of inhibition is indicative of network maturation state and that the PNNs may stabilize the network and limit plasticity.

Furthermore, reduced inhibitory activity after PNN removal may be of relevance for several neurological diseases in which dysfunction of inhibitory PV + neurons have been implicated, most notably schizophrenia (Lewis et al., 2012). Interestingly, postmortem studies of schizophrenia patients indicate that PNNs are depleted (Berretta, 2012; Pantazopoulos et al., 2015). Another hallmark of schizophrenia thought to arise from alterations in $\mathrm{PV}+$ neuron function is changes to the LFP oscillations. Oscillations in the gamma-frequency range result from synchronized activity of PV + neurons (Cardin et al., 2009). Given the reduced activity of inhibitory neurons after PNN removal, we investigated how this affected oscillations of the LFP in the gamma-frequency range. We found that the PNN-depleted animals were very similar to controls (Fig. 4). The chABC-treated animals had significantly higher power in the low-gamma range during spontaneous activity, accompanied by higher theta power, indicating that the basic network function may be altered. We found that both groups of animals had sparse gamma oscillations compared with previous recordings from head-fixed mice (Niell and Stryker, 2010). The difference could be due to differences in the experimental setup or differences between species (Buzsáki et al., 2003; Niell and Stryker, 2010). Although the overall gamma activity in our data is low, the lack of gamma in response to sensory stimuli corresponds with recent work in which gamma power in response to neutral stimuli is lower than during spontaneous activity (Chen et al., 2015). Stronger gamma oscillations may be expected if the animal is alert, such as during an associative learning task (Igarashi et al., 2014). Gamma oscillations have been correlated with sensory stimulation in anesthetized animals, locomotion, attention, and memory load (Niell and Stryker, 2008, 2010; Igarashi et al., 2014; Pritchett et al., 2015). Therefore, future investigations to reveal the role PNNs in network processing should be conducted in experiments for which gamma activity is expected to play a role.

To study plasticity, we used MD. Five days of eye closure in chABC-treated rats was sufficient to produce a shift in ODI in favor of the nondeprived eye similar to that of juvenile animals (Fagiolini et al., 1994; Guire et al., 1999; Deidda et al., 2015). Although OD plasticity has been the canonical model to study activity-dependent plasticity for decades, the underlying mechanisms and time course of events remain unresolved (Kuhlman et al., 2013; Cooke and Bear, 2014). We used chronic recordings to monitor the very same units during the first $48 \mathrm{~h}$ of MD (Fig. 6). This is one of very few investigations to monitor the activity of the same units in the binocular region of V1 during OD plasticity (Mioche and Singer, 1989; Rose et al., 2016). Whereas Hengen et al. (2013) conducted a similar experiment, they recorded from the monocular part of $\mathrm{V} 1$ and the most prominent plasticity changes during OD plasticity occur in the binocular part. As expected, the unit responses in adult controls showed no difference from baseline recordings, although we observed large variability in the response patterns. In contrast, units recorded from chABC-treated animals showed a significant reduction in activity 
after contralateral eye closure after just $12 \mathrm{~h}$. This suggests very rapid plasticity mechanisms and was accompanied by a somewhat slower potentiation of responses in neurons ipsilateral to the deprived eye. In contrast to all other recordings, the recordings to assess OD were performed in anesthetized animals. Anesthesia is known to affect correlated activity and the activity of both inhibitory and excitatory units (Vizuete et al., 2012; Goltstein et al., 2015). However, the effect of anesthesia on spiking activity is stronger during spontaneous activity compared with stimulus-evoked firing, for which behavioral state has a stronger impact (Niell and Stryker, 2010). We cannot infer directly that the changes in unit activity observed during MD in awake recordings correspond directly to recordings performed under anesthesia after MD. However, recordings to assess ODI in head-fixed, awake animals give highly comparable results to those performed in anesthesia (Frenkel and Bear, 2004; Rose et al., 2016). Given the profoundly different responses of units and LFP in chABCtreated rats compared with controls in the first hours of $\mathrm{MD}$, we believe that the changes in unit activity represent the early stages of plasticity that eventually give rise to changes in OD. Together, our data from chronically recorded single units are similar to previous work on $\mathrm{CP}$ aged animals looking at population activity alone, but the changes in activity occur earlier in our data (Frenkel and Bear, 2004; Gandhi et al., 2008; but see Kuhlman et al., 2013). We provide evidence indicating that PNN removal opens for plasticity by similar mechanisms to those of OD plasticity during the CP. However, the neuronal circuitry of the juvenile brain is likely different from resetting the circuitry of adult brain, likely also affecting the mechanisms of OD plasticity. This will need further investigations in, for example, genetically modified animals in which PNNs never form.

Closing one eye caused dramatic effects in the LFP, giving rise to highly synchronized gamma oscillations in chABC-treated animals, but not in controls (Fig. 7). A similar effect was seen with $\mathrm{MD}$ in $\mathrm{CP}$ aged animals (Fig. 8), suggesting that this may be a common feature of circuits of high plasticity when load is placed on the network, such as during the initial phase of sensory deprivation. Supporting this hypothesis is the finding that synchronized gamma activity is strongly correlated with learning in the highly plastic hippocampus (Igarashi et al., 2014). Similarly, mismatch in input from the eyes at the start of MD may activate ensembles of these cells in a highly synchronous manner, giving rise to strong gamma oscillations driving plasticity processes. The functional role of PNNs in this process may be to restrict the PV+ network and thereby restrict plasticity.

\section{References}

Balmer TS, Carels VM, Frisch JL, Nick TA (2009) Modulation of perineuronal nets and parvalbumin with developmental song learning. J Neurosci 29:12878-12885. CrossRef Medline

Barthó P, Hirase H, Monconduit L, Zugaro M, Harris KD, Buzsáki G (2004) Characterization of neocortical principal cells and interneurons by network interactions and extracellular features. J Neurophysiol 92:600-608. CrossRef Medline

Bereshpolova Y, Stoelzel CR, Zhuang J, Amitai Y, Alonso JM, Swadlow HA (2011) Getting drowsy? Alert/non-alert transitions and visual thalamocortical network dynamics. J Neurosci 31:17480-17487. CrossRef Medline

Berretta S (2012) Extracellular matrix abnormalities in schizophrenia. Neuropharmacology 62:1584-1597. CrossRef Medline

Beurdeley M, Spatazza J, Lee HH, Sugiyama S, Bernard C, Di Nardo AA, Hensch TK, Prochiantz A (2012) Otx2 binding to perineuronal nets persistently regulates plasticity in the mature visual cortex. J Neurosci 32:9429-9437. CrossRef Medline

Brainard DH (1997) The Psychophysics Toolbox. Spat Vis 10:433-436. CrossRef Medline
Brückner G, Brauer K, Härtig W, Wolff JR, Rickmann MJ, Derouiche A, Delpech B, Girard N, Oertel WH, Reichenbach A (1993) Perineuronal nets provide a polyanionic, glia-associated form of microenvironment around certain neurons in many parts of the rat brain. Glia 8:183-200. CrossRef Medline

Brückner G, Bringmann A, Härtig W, Köppe G, Delpech B, Brauer K (1998) Acute and long-lasting changes in extracellular-matrix chondroitinsulphate proteoglycans by injection of chondroitinase $\mathrm{ABC}$ in the adult rat brain. Exp Brain Res 121:300-310. CrossRef Medline

Brunel N (2000) Dynamics of sparsely connected networks of excitatory and inhibitory spiking neurons. J Comput Neurosci 8:183-208. CrossRef Medline

Bruno RM, Simons DJ (2002) Feedforward mechanisms of excitatory and inhibitory cortical receptive fields. J Neurosci 22:10966-10975. Medline

Buzsáki G, Buhl DL, Harris KD, Csicsvari J, Czéh B, Morozov A (2003) Hippocampal network patterns of activity in the mouse. Neuroscience 116:201-211. CrossRef Medline

Cabungcal JH, Steullet P, Morishita H, Kraftsik R, Cuenod M, Hensch TK, Do KQ (2013) Perineuronal nets protect fast-spiking interneurons against oxidative stress. Proc Natl Acad Sci U S A 110:9130-9135. CrossRef Medline

Cardin JA, Carlén M, Meletis K, Knoblich U, Zhang F, Deisseroth K, Tsai LH, Moore CI (2009) Driving fast-spiking cells induces gamma rhythm and controls sensory responses. Nature 459:663-667. CrossRef Medline

Carulli D, Pizzorusso T, Kwok JC, Putignano E, Poli A, Forostyak S, Andrews MR, Deepa SS, Glant TT, Fawcett JW (2010) Animals lacking link protein have attenuated perineuronal nets and persistent plasticity. Brain 133:2331-2347. CrossRef Medline

Chen G, Rasch MJ, Wang R, Zhang XH (2015) Experience-dependent emergence of beta and gamma band oscillations in the primary visual cortex during the critical period. Sci Rep 5:17847. CrossRef Medline

Colgin LL, Denninger T, Fyhn M, Hafting T, Bonnevie T, Jensen O, Moser MB, Moser EI (2009) Frequency of gamma oscillations routes flow of information in the hippocampus. Nature 462:353-357. CrossRef

Cooke SF, Bear MF (2014) How the mechanisms of long-term synaptic potentiation and depression serve experience-dependent plasticity in primary visual cortex. Philos Trans R Soc Lond B Biol Sci 369:20130284. CrossRef Medline

Deidda G, Allegra M, Cerri C, Naskar S, Bony G, Zunino G, Bozzi Y, Caleo M, Cancedda L (2015) Early depolarixing GABA controls critical period plasticity in rat visual cortex. Nat Neurosci 18:87-96. CrossRef Medline

Donato F, Rompani SB, Caroni P (2013) Parvalbumin-expressing basketcell network plasticity induced by experience regulates adult learning. Nature 504:272-276. CrossRef Medline

Fagiolini M, Hensch TK (2000) Inhibitory threshold for critical-period activation in primary visual cortex. Nature 404:183-186. CrossRef Medline

Fagiolini M, Pizzorusso T, Berardi N, Domenici L, Maffei L (1994) Functional development of the rat primary visual cortex and the role of visual experience: dark rearing and monocular deprivation. Vision Res 34709 34720. Medline

Fagiolini M, Fritschy JM, Löw K, Möhler H, Rudolph U, Hensch TK (2004) Specific GABAA circuits for visual cortical plasticity. Science 303:16811683. Medline

Frenkel MY, Bear MF (2004) How monocular deprivation shifts ocular dominance in visual cortex of young mice. Neuron 44:917-923. CrossRef Medline

Gandhi SP, Yanagawa Y, Stryker MP (2008) Delayed plasticity of inhibitory neurons in developing visual cortex. Proc Natl Acad Sci U S A 105:1679716802. CrossRef Medline

Gewaltig MO, Diesmann M (2007) NEST (Neural Simulation Tool). Scholarpedia 2:1430. CrossRef

Girman SV, Sauvé Y, Lund RD (1999) Receptive field properties of single neurons in rat primary visual cortex. J Neurophysiol 82:301-311. Medline

Gogolla N, Caroni P, Lüthi A, Herry C (2009) Perineuronal nets protect fear memories from erasure. Science 325:1258-1261. CrossRef Medline

Goltstein PM, Montijn JS, Pennartz CM (2015) Effects of isoflurane anesthesia on ensemble patterns of Ca2 + activity in mouse V1: Reduced direction selectivity independent of increased correlations in cellular activity. PLoS One 10:e0118277. CrossRef Medline

Guire ES, Lickey ME, Gordon B (1999) Critical period for the monocular deprivation effect in rats: Assessment with sweep visually evoked potentials. J Neurophysiol 81:121-128. Medline 
Haggerty DC and Ji D (2015) Activities of visual cortical and hippocampal neurons co-fluctuate in freely moving rats during spatial behavior. eLife 4:e08902. CrossRef Medline

Happel MF, Niekisch H, Castiblanco Rivera LL, Ohl FW, Deliano M, Frischknecht R (2014) Enhanced cognitive flexibility in reversal learning induced by removal of the extracellular matrix in auditory cortex. Proc Natl Acad Sci U S A 111:2800-2805. CrossRef Medline

Härtig W, Brauer K, Brückner G (1992) Wisteria floribunda agglutininlabeled nets surround parvalbumin-containing neurons. Neuroreport 3:869-872. CrossRef Medline

Härtig W, Derouiche A, Welt K, Brauer K, Grosche J, Mäder M, Reichenbach A, Brückner G (1999) Cortical neurons immunoreactive for the potassium channel Kv3.1b subunit are predominantly surrounded by perineuronal nets presumed as a buffering system for cations. Brain Res 842: 15-29. CrossRef Medline

Hengen KB, Lambo ME, Van Hooser SD, Katz DB, Turrigiano GG (2013) Firing rate homeostasis in visual cortex of freely behaving rodents. Neuron 80:335-342. CrossRef Medline

Hensch TK (2005) Critical period plasticity in local cortical circuits. Nat Rev Neurosci 6:877-888. Medline

Hensch TK, Fagiolini M, Mataga N, Stryker MP, Baekkeskov S, Kash SF (1998) Local GABA circuit control of experience-dependent plasticity in developing visual cortex. Science 282:1504-1508. CrossRef Medline

Hockfield S, Kalb RG, Zaremba S, Fryer H (1990) Expression of neural proteoglycans correlates with the acquisition of mature neuronal properties in the mammalian brain. Cold Spring Harb Symp Quant Biol 55:505-514. CrossRef Medline

Igarashi KM, Lu L, Colgin LL, Moser MB, Moser EI (2014) Coordination of entorhinal-hippocampal ensemble activity during associative learning. Nature 510:143-147. CrossRef Medline

Ji D, Wilson MA (2007) Coordinated memory replay in the visual cortex and hippocampus during sleep. Nat Neurosci 10:100-107. CrossRef Medline

Kuhlman SJ, Olivas ND, Tring E, Ikrar T, Xu X, Trachtenberg JT (2013) A disinhibitory microcircuit initiates critical-period plasticity in the visual cortex. Nature 501:543-546. CrossRef Medline

Kwok JC, Afshari F, García-Alías G, Fawcett JW (2008) Proteoglycans in the central nervous system: plasticity, regeneration and their stimulation with Chondroitinase ABC. Restor Neurol Neurosci 26:131-145. Medline

Levelt CN, Hübener M (2012) Critical-period plasticity in the visual cortex. Annu Rev Neurosci 35:309-330. CrossRef Medline

Lewis DA, Curley AA, Glausier JR, Volk DW (2012) Cortical parvalbumin interneurons and cognitive dysfunction in schizophrenia. Trends Neurosci 35:57-67. CrossRef Medline

Liu H, Gao PF, Xu HW, Liu MM, Yu T, Yao JP, Yin ZQ (2013) Perineuronal nets increase inhibitory GABAergic currents during the critical period in rats. Int J Ophthalmol 6:120-125. CrossRef Medline

Markram H, Toledo-Rodriguez M, Wang Y, Gupta A, Silberberg G, Wu C (2004) Interneurons of the neocortical inhibitory system. Nat Rev Neurosci 5:793-807. CrossRef Medline

Medini P (2011) Layer -and -cell-type-specific subthreshold and suprathreshold effects of long-term monocular deprivation in rat visual cortex. J Neurosci 31:17134-17148. CrossRef Medline
Mioche L, Singer W (1989) Chronic recordings from single sites of kitten striate cortex during experience-dependent modifications of receptivefield properties. J Neurophysiol 62:185-197. Medline

Morawski M, Reinert T, Meyer-Klaucke W, Wagner FE, Tröger W, Reinert A, Jäger C, Brückner G, Arendt T (2015) Ion exchanger in the brain: quantitative analysis of perineurally fixed anionic binding sites suggests diffusion barriers with ion sorting properties. Sci Rep 5:16471. CrossRef Medline

Niell CM, Stryker MP (2008) Highly selective receptive fields in mouse visual cortex. J Neurosci 28:7520-7536. CrossRef Medline

Niell CM, Stryker MP (2010) Modulation of visual responses by behavioral state in mouse visual cortex. Neuron 65:472-479. CrossRef Medline

Pantazopoulos H, Markota M, Jaquet F, Ghosh D, Wallin A, Santos A, Caterson B, Berretta S (2015) Aggrecan and chondroitin-6-sulfate abnormalities in schizophrenia and bipolar disorder: a postmortem study on the amygdala. Transl Psychiatry 5:e496. CrossRef Medline

Paxinos G, Watson C (2007) The rat brain in stereotaxic coordinates, Ed 6 . San Diego: Academic.

Pizzorusso T, Medini P, Berardi N, Chierzi S, Fawcett JW, Maffei L (2002) Reactivation of ocular dominance plasticity in the adult visual cortex. Science 298:1248-1251. CrossRef Medline

Pritchett DL, Siegle JH, Deister CA, Moore CI (2015) For things needing your attention: the role of neocortical gamma in sensory perception. Curr Opin Neurobiol 31:254-263. CrossRef Medline

Rose T, Jaepel J, Hübener M, Bonhoeffer T (2016) Cell-specific restoration of stimulus preference after monocular deprivation in the visual cortex. Science 352:1319-1322. CrossRef Medline

Sohal VS, Zhang F, Yizhar O, Deisseroth K (2009) Parvalbumin neurons and gamma rhythms enhance cortical circuit performance. Nature 459: 698-702. CrossRef Medline

Southwell DG, Froemke RC, Alvarez-Buylla A, Stryker MP, Gandhi SP (2010) Cortical plasticity induced by inhibitory neuron transplantation. Science 327:1145-1148. CrossRef Medline

Toyoizumi T, Miyamoto H, Yazaki-Sugiyama Y, Atapour N, Hensch TK, Miller KD (2013) A theory of the transition to critical period plasticity: inhibition selectively suppresses spontaneous activity. Neuron 80:51-63. CrossRef Medline

Vizuete JA, Pillay S, Diba K, Ropella KM, Hudetz AG (2012) Monosynaptic functional connectivity in cerebral cortex during wakefulness and under graded levels of anesthesia. Front Integr Neurosci 6:90. CrossRef Medline

Wallace DJ, Greenberg DS, Sawinski J, Rulla S, Notaro G, Kerr JN (2013) Rats maintain an overhead binocular field at the expense of constant fusion. Nature 498:65-69. CrossRef Medline

Wang XD, Chen C, Zhang D, Yao H (2014) Cumulative latency advance underlies fast visual processing in desynchronized brain state. Proc Natl Acad Sci U S A 111:515-520. CrossRef Medline

Wiesel TN, Hubel DH (1963) Single-cell responses in striate cortex of kittens deprived of vision in one eye. J Neurophysiol 26:1003-1017. Medline

Zhuang J, Bereshpolova Y, Stoelzel CR, Huff JM, Hei X, Alonso JM, Swadlow HA (2014) Brain state effects layer 4 of the awake visual cortex. J Neurosci 34:3888-3900. CrossRef Medline 